\title{
The AKT kinase signaling network is rewired by PTEN to control proximal BCR signaling in germinal center B cells
}

\author{
Wei Luo ${ }^{1,2}$, William Hawse ${ }^{1,2}$, Laura Conter ${ }^{1}$, Nikita Trivedi ${ }^{1}$, Florian Weisel ${ }^{1}$, Daniel \\ Wikenheiser $^{1}$, Richard T. Cattley ${ }^{1}$, Mark J. Shlomchik ${ }^{1}$ \\ 1.Department of Immunology, School of Medicine, University of Pittsburgh \\ 2.These authors contributed equally
}

\begin{abstract}
Compared to naïve B cells (NBCs), both B cell antigen receptor (BCR) and CD40 signaling are rewired in germinal center (GC) B cells (GCBCs) to optimize selection for high-affinity B cells. The mechanism for $\mathrm{BCR}$ reprogramming in GCBCs remains unknown. We describe a GCspecific, AKT kinase-driven negative feedback loop that attenuates BCR signaling. A mass spectrometry proteomic approach revealed that AKT activity was retargeted in GCBCs compared to NBCs. Retargeting was linked to differential AKT T308 and S473 phosphorylation, in turn due to GC-specific upregulation of phosphoinositide-dependent protein kinase PDK1 and the phosphatase PTEN, which retuned phosphatidylinositol-3-OH kinase (PI3K) signals. In GCBCs, AKT preferentially targeted CSK, SHP-1 and HPK1, which are negative regulators of BCR signaling. Phosphorylation results in markedly increased enzymatic activity of these proteins, creating a negative-feedback loop that dampens upstream BCR signaling. Inhibiting AKT substantially enhanced activation of BCR proximal kinase LYN as well as downstream BCR signaling molecules in GCBCs, establishing the relevance of this pathway.
\end{abstract}

\section{Introduction}

\begin{abstract}
Signaling pathways translate external cues to appropriate cellular responses. In lymphocytes, signaling has largely been studied in naïve or resting states in order to determine how signals lead to initial activation, clonal expansion and differentiation. How signal interpretation is remodeled in responding cells is poorly studied.

GCBCs are exceptional among activated lymphocytes in that, once GCs reach peak size, they undergo neither expansion in net cell number nor effector differentiation. Instead, the $\mathrm{GC}$ reaction reaches a steady-state number of GCBCs, with proliferation balanced by cell death, engendering intense clonal selection ${ }^{1,2,3}$. Further, during the GC reaction, small
\end{abstract}

Users may view, print, copy, and download text and data-mine the content in such documents, for the purposes of academic research, subject always to the full Conditions of use:http://www.nature.com/authors/editorial_policies/license.html\#terms

Author Contributions

W.L., W.H., and M.J.S. designed research, interpreted data, and wrote the manuscript. W.L., W.H., L.C., N.T., F.W., D.W., and R.T.C. did the experiments and analyzed the data.

Competing Interests Statement

The authors declare no competing interests. 
numbers of long-lived memory B and plasma cells are differentiated ${ }^{4}$. To accomplish these tasks, it is likely that GCBCs must be reprogrammed to a "third state" that is distinct from either naïve or effector-activated B cells. Indeed, by expressing various transcriptional regulators, most notably the transcription factor B-cell lymphoma 6 protein (Bcl-6), GCBCs alter expression of a large number of genes and remodel their epigenome ${ }^{5,6,7}$. As a consequence, it is reasonable to think that $\mathrm{B}$ cell signal interpretation is also rewired during the GC reaction.

Our lab and others have been studying how GCBCs respond to environmental cues differently from other B cell lineages. These signals include adhesion and cell-cell interacting molecules, cytokines, and antigen $8,910,11,1213$. We have focused on how signals that drive antigen selection are interpreted differently in GCBCs compared to other B cells. In NBCs, BCR signals trigger the phosphorylation of Iga $\beta$ immunoreceptor tyrosine activation motifs (ITAMs) by the Src-family kinase LYN, leading to the activation of the kinase SYK. These events initiate the signalosome formation and the activation of multiple downstream pathways ${ }^{14}$. Most notably, we found that BCR signals are markedly attenuated and qualitatively altered in GCBCs compared to $\mathrm{NBCs}^{15}, 16$. SYK kinase phosphorylation is much reduced, resulting in very little downstream activation of the transcription factor NF$\kappa \mathrm{B}$. The PI3K-AKT signaling pathway is also altered, with reduced generation of p-S473 AKT and downstream kinase target p-S6, yet with strong phosphorylation of the AKT target FOXO1 transcription factor, which plays a critical role in antigen-driven GCBC selection ${ }^{16,17,18}$. We recently showed that this limited BCR-mediated downstream signal must cooperate with CD40 signals-which are also rewired to attenuate PI3K but maintain $\mathrm{NF}-\mathrm{\kappa B}$ transduction - to synergistically induce c-MYC and support positive selection of

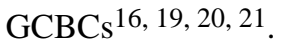

Although attenuation of BCR signaling in GCBCs is critical for survival and selection of cells in this site, little is known about the mechanisms by which GCBCs actually rewire their BCR signaling apparatus. We previously implicated increased phosphatase activity in this process, and obtained evidence that both SHP-1 and SHIP-1 were potentially more active in $\mathrm{GCBCs}^{15}$. However, beyond this, the specific mechanisms for BCR signal attenuation and remodeling of PI3K-AKT signaling have not been elucidated.

Here, we identify a GC-specific AKT signaling network and demonstrate that it functions in a negative-feedback loop to activate negative regulators of upstream BCR signaling. These studies also revealed several novel targets of AKT that are enzymes and show that the phosphorylation of these yields increased enzymatic activity. We further demonstrate how AKT signaling is differentially targeted in GCBCs vs. NBCs, which is due at least in part to GC-specific modulation of PI3K signals. We found that GCBCs express high amounts of PTEN, which leads to reduced abundance of phosphatidylinositol-(3,4,5)-trisphosphate $\left(\operatorname{PtdIns}(3,4,5) \mathrm{P}_{3}\right)$ but increased phosphatidylinositol-(4,5)-bisphosphate $\left(\operatorname{Ptd} \operatorname{Ins}(4,5) \mathrm{P}_{2}\right)$ generation upon BCR ligation. Nonetheless, GCBCs express high amounts of PDK1, which can sensitively detect PtdIns $(3,4,5) \mathrm{P}_{3}$. Combined, these features result in robust AKT T308 phosphorylation but attenuated S473 phosphorylation, leading to generation of a GCspecific AKT target profile. These studies thus provide insights into both PI3K-AKT 
signaling biology as well the mechanism by which GCBCs retune BCR signaling to promote affinity selection.

\section{Results}

\section{Phosphorylation of AKT is altered in GCBCs}

We previously showed that signals regulating FOXO1 and the metabolic checkpoint kinase mTORC1 are rewired in GCBCs compared to NBCs ${ }^{16}$. Since both FOXO1 and mTORC1 are regulated by AKT, we hypothesized that AKT signaling in GCBCs would be reprogramed to alter these downstream pathways. We previously found that AKT S473 was poorly phosphorylated in both unstimulated NBCs and GCBCs ${ }^{16}$. Here we found that, in cells isolated directly ex vivo, total AKT protein amounts were elevated in GCBCs and that AKT T308 was naturally phosphorylated to a greater extent in GCBCs than in NBCs (Fig. 1a,b and Supplementary Fig. 1a,b). PDK1 is the upstream kinase for AKT T308 phosphorylation, and phosphorylation of PDK1 at S241 is essential for its kinase activity 22 . We found that both total and S241 phosphorylated PDK1 were more abundant in GCBCs compared to NBCs (Fig. 1c). As expected from our prior report ${ }^{16}$, AKT S473 phosphorylation was attenuated and transient in GCBCs upon BCR stimulation (Fig. 1d). In contrast, BCR signaling induced robust AKT T308 phosphorylation in GCBCs, comparable to that seen in NBCs (Fig. 1d), despite the fact that BCR signaling in general is markedly attenuated in the GCBCs ${ }^{15,16}$ (Fig. 1e). These findings suggest that elevated PDK1 expression and activity in GCBCs might compensate for an attenuated upstream signal to enable efficient AKT p-T308 generation.

\section{Higher PTEN restrains AKT S473 phosphorylation in GCBCs}

We then asked why AKT S473 phosphorylation in GCBCs is dampened. AKT S473 phosphorylation is catalyzed by mTORC2, which has been shown to have a markedly lower affinity for PtdIns(3,4,5)P3 compared to PDK123, 24, 25, 26. We hypothesized that PtdIns $(3,4,5) \mathrm{P}_{3}$ generation in GCBCs is controlled to allow efficient activation of PDK1 but not mTORC2. PtdIns $(3,4,5) \mathrm{P}_{3}$ generation requires $\mathrm{PI} 3 \mathrm{~K}$, and in $\mathrm{B}$ cells the catalytic subunit p1108 is the major isoform mediating antigen-dependent BCR signaling ${ }^{27}$. We found that GCBCs expressed more, not less, p110反 protein than NBCs (Supplementary Fig. 2), suggesting the rewired signaling in GCBCs is not due to reduced expression of the catalytic subunit of PI3K. In contrast, PTEN, which negatively regulates AKT activation by converting PtdIns $(3,4,5) \mathrm{P}_{3}$ to PtdIns $(4,5) \mathrm{P}_{2}{ }^{28}$, was more highly expressed in GCBCs than in NBCs (Fig. 2a). The finding of elevated PTEN protein in GCBCs suggested that the balance between PtdIns $(4,5) \mathrm{P}_{2}$ and PtdIns $(3,4,5) \mathrm{P}_{3}$ might differ between NBCs and GCBCs. To test this, generation of PtdIns $(4,5) \mathrm{P}_{2}$ and $\operatorname{PtdIns}(3,4,5) \mathrm{P}_{3}$ was measured as a function of time after BCR ligation. Compared to NBC, GCBCs generated less PtdIns $(3,4,5) \mathrm{P}_{3}$, which returned to baseline within 5 min or less, yet GCBCs accumulated more PtdIns $(4,5) \mathrm{P}_{2}$ (Fig. $2 b$ ), in keeping with higher PTEN amounts in GCBCs. The kinetics of PtdIns $(3,4,5) \mathrm{P}_{3}$ generation upon BCR ligation correlated very well with attenuated BCR signaling and AKT S473 phosphorylation (Fig. 1d,e and Fig. 2b), further suggesting that high PTEN expression in GCBCs might restrain AKT S473 phosphorylation by inhibiting PtdIns $(3,4,5) \mathrm{P}_{3}$ generation and favoring generation of $\operatorname{PtdIns}(4,5) \mathrm{P}_{2}$. To test this, we used the PTEN 
inhibitor SF1670. Acute inhibition of PTEN significantly increased PtdIns $(3,4,5) \mathrm{P}_{3}$ and PtdIns(3,4,) $\mathrm{P}_{2}$ generation in GCBCs upon BCR stimulation, while, it resulted in a significant decrease in the PTEN-dependent product of $\operatorname{PtdIns}(3,4,5) \mathrm{P}_{3}$ hydrolysis, $\operatorname{PtdIns}(4,5) \mathrm{P}_{2}$ (Fig. 2c). These results were commensurate with our previous finding that SHIP-1 activity is elevated in GCBCs ${ }^{15}$; SHIP-1 could limit the accumulation of $\operatorname{PtdIns}(3,4,5) \mathrm{P}_{3}$ by converting PtdIns(3,4,5) $\mathrm{P}_{3}$ into PtdIns(3,4,) $\mathrm{P}_{2}$. More importantly, pretreatment with SF1670 increased BCR signal-induced AKT S473 phosphorylation in GCBCs (Fig. 2d), which is dependent on PtdIns $(3,4,5) \mathrm{P}_{3}$ stimulation of mTORC2 activity. PTEN inhibition also increased p-S6 in GCBCs (Fig. 2d). These findings indicate that suppression of the AKT-mTORC1-S6 axis is mechanistically linked to high PTEN activity in GCBCs.

\section{AKT targets different pathways in GCBCs compared to NBCs}

Previous work demonstrated that differential phosphorylation regulates AKT substrate specificity $29,30,31$. Since GCBCs and NBC differ in AKT p-T308 and p-S473, we asked whether AKT substrates differ between NBCs and GCBCs. Immunoblotting using an AKT phospho-substrate antibody that specifically recognizes the phosphorylated AKT substrate motif (RXRXXS*/T*) showed that the substrates of AKT in GCBCs qualitatively differed from NBCs as well as activated non-GCBCs (Fig. 3a and Supplementary Fig. 1b).

To obtain a deeper understanding of the GCBC-specific AKT signaling network, we used beads coated with AKT phospho-substrate antibody to immunoprecipitate AKTphosphorylated substrates from lysates derived from fresh or BCR-stimulated NBCs and GCBCs, followed by a quantitative mass spectrometric assay (Supplementary Fig. 3 and Supplementary Table 1). We identified 33 unique AKT substrates in BCR-activated NBCs and 63 in activated GCBCs, with other patterns of substrate sharing depicted in the Venn diagram (Fig. 3b). In these cell type-specific AKT substrates, we found that AKT preferentially phosphorylated proteins involved in regulating the actin cytoskeleton in NBCs (Fig. 3c). Interestingly, in GCBCs AKT preferentially phosphorylated proteins with functions related to cell control, including transcriptional regulation, RNA processing and signal transduction (Fig. 3c). Together, these results demonstrate that the AKT kinase signaling network is rewired in GCBCs compared to NBCs.

\section{AKT targets proximal BCR signaling regulators in GCBCs}

Given that BCR signaling is dampened in $\mathrm{GCBCs}{ }^{15}, 16,32,33$, it was interesting that GCBCspecific AKT targets included negative regulators of BCR signaling, most notably CSK, SHP-1 and HPK1 (Fig. 4a). To evaluate the expression of these three proteins, along with other relevant proteins in the $\mathrm{BCR} / \mathrm{PI} 3 \mathrm{~K}$ signaling cascade, we performed immunoblot analysis with cell lysates of NBCs and GCBCs isolated directly ex vivo (Supplementary Fig. $1 \mathrm{~b}$ and Supplementary Fig.2). We found that total protein amounts of CSK and HPK1 were higher in GCBCs than NBCs (Supplementary Fig. 2). CSK is the major kinase for the inhibitory tyrosine of LYN, which when phosphorylated maintains LYN in an inactive conformation $^{34}$. SHP-1 is a phosphatase for multiple activating P-Tyr modifications, including on SYK and BLNK ${ }^{35}$. HPK1 is a kinase for BLNK, the phosphorylation of which by HPK1 inhibits its linker activity for BCR signal transduction ${ }^{36}$. The proteomics screen 
also identified that ARP2, a subunit in the ARP2/3 complex that regulates actin polymerization ${ }^{37}$, was phosphorylated by AKT in activated NBCs but not GCBCs (Fig. 4a).

To confirm that these proteins were AKT substrates, NBCs and GCBCs were BCRstimulated with or without an AKT inhibitor, and immunoblotting was used to detect proteins that were immunoprecipitated by the AKT phospho-substrate antibody (Fig. 4b). In agreement with the mass spectrometry results, CSK, SHP-1 and HPK1 were immunoprecipitated by this antibody only in GCBCs, validating these proteins as GCBCsspecific AKT substrates (Fig. 4b). Likely due the different nature of the assays, AKTdependent phosphorylation of SHP-1 and HPK1 was only found in stimulated GCBCs by immunoblotting, whereas the mass spectrometric screening detected it in stimulated as well as unstimulated GCBCs. Immunoblotting detected ARP2 as an AKT substrate in NBCs only (Fig. 4b), again consistent with the mass spectrometry assay. These results were validated by AKT inhibition, which blocked immunoprecipitation of all these proteins (Fig. $4 \mathrm{~b}$ right lanes). We included LYN as a negative control, which was not identified by mass spectrometry screening. As expected LYN was not immunoprecipitated by the AKT phospho-substrate antibody under any of the conditions tested (Fig. 4b).

To further confirm results from the mass spectrometry assay, we performed in vitro kinase assays with recombinant activated AKT and found that AKT could effectively phosphorylate recombinant CSK, SHP-1 and HPK1 when ATP was present (Fig. 4c). As a negative control, recombinant AKT did not phosphorylate recombinant LYN in vitro. Together, our mass spectrometry screening and validation experiments suggest that AKT specifically phosphorylates at least three negative regulators of BCR signaling in GCBCs but not NBCs.

\section{AKT phosphorylation enhances negative regulator activity}

Next we used mass spectrometry to identify potential AKT phosphorylation sites on CSK, SHP-1 and HPK1, followed by functional assays to determine the consequences of phosphorylation. CSK was phosphorylated by AKT at S284 (Fig. 5a). Phosphorylation of S284 was modeled onto the CSK-c-SRC crystal structure ${ }^{38}$, which suggested that phosphorylation would enhance the binding between CSK and its substrate and thus might increase CSK kinase activity on LYN and other Src family kinases. We found that recombinant CSK that had been phosphorylated by AKT indeed had a seven-fold higher rate for phosphorylating LYN at Y507 than unphosphorylated CSK (Fig. 5b). AKT phosphorylated SHP-1 on T394, which is within the catalytic domain (Fig. 5c), suggesting functional impact. In fact, enzymatic activity of AKT-phosphorylated SHP-1 was increased by five-fold compared to unmodified SHP-1 (Fig. 5d). This is also consistent with reported increased SHP-1 tyrosine phosphatase activity in GCBCs ${ }^{15}$. HPK1 phosphorylates BLNK on T152, which down-modulates BCR signaling ${ }^{36}$. However, the effect of phosphorylation of HPK1 by AKT has not been documented. We found that AKT-catalyzed phosphorylation sharply increased the activity of HPK1 for phosphorylating BLNK (Fig. 5e). Hence, in each case, AKT-dependent phosphorylation increased the enzymatic activity of negative regulators of BCR signaling. 


\section{PTEN inhibition alters AKT signaling networks in GCBCs}

To investigate whether elevated PTEN expression in GCBCs is essential for maintaining the GCBC-specific AKT signaling network, we pretreated GCBCs or NBCs with PTEN inhibitor (SF1670) or DMSO before stimulation. We then immunoprecipitated AKT phospho-substrates from cell lysates and performed quantitative mass spectrometric analysis (Supplementary Fig. 3 and Supplementary Table 2). A PANTHER pathway analysis demonstrated that PTEN inhibition substantially altered AKT signaling networks in GCBCs, with ontogenies of signaling, transcription and RNA processing manifesting major effects (Fig. 6a and Supplementary Fig. 4). Specifically, PTEN inhibition reduced the level of AKT phosphorylation of CSK, SHP1 and HPK1 in GCBCs (Fig. 6a,b). On the other hand, AKTcatalyzed ARP2 phosphorylation was increased by PTEN inhibition in GCBCs (Fig. 6a,b). These data link GCBC-specific alterations observed in AKT substrates to PTEN-dependent regulation of phosphatidylinositol phosphate species generation.

\section{AKT inhibition enhances proximal BCR signaling in GCBCs}

The proteomic and in vitro kinase assays presented above indicated that AKT in GCBCs could enhance at least three important negative regulators of BCR signaling. We therefore hypothesized that AKT activity in GCBCs negatively controls upstream BCR signaling. The hypothesis predicts that an AKT specific inhibitor would enhance the response to BCR stimulation specifically in GCBCs. Remarkably, AKT inhibition significantly enhanced the amount of active LYN in GCBCs, as measured by the ratio of activating-to-inhibitory tyrosine phosphorylation (Y396/Y507) ${ }^{34,39}$ (Fig. 7a). The overall phosphorylation of SYK is a reflection of both LYN activity and SHP-1-mediated phosphatase activity. Our results and model predicted that AKT inhibition would lead to reduced activities of CSK and SHP-1, in turn leading to more LYN kinase activity on SYK as well as less phosphatase activity to hydrolyze $\mathrm{p}-\mathrm{SYK}$. Consistent with this, AKT inhibition resulted in notably higher and more persistent amounts of p-SYK in BCR-stimulated GCBCs (Fig. 7a). To indirectly assess the functional effects of AKT inhibition on HPK1, and the resulting effects on generation of signals downstream of BLNK, we measured the generation of both p-BTK and $\mathrm{p}-\mathrm{PLC} \gamma 2$. Both were increased by AKT inhibition at all time points after one minute of stimulation (Fig. 7b). Importantly, this effect was GCBC-specific since AKT inhibition did not significantly change the kinetics of p-BTK or p-PLC $\gamma 2$ in NBCs (Supplementary Fig. 5). These data thus reveal a novel mechanism by which AKT signaling in GCBCs is rewired to form a negative feedback loop acting on proximal BCR signaling and further demonstrate how this operates at multiple levels of the signaling pathway.

\section{Discussion}

We have identified molecular mechanisms behind a novel feedback loop in which AKT signaling phosphorylates - and thereby activates - three distinct upstream negative regulators of BCR signaling. This negative feedback loop is found only in GCBCs, not NBCs, presumably because AKT targeting itself is redirected within GCBCs. We further linked the redirection of AKT target specificity in GCBCs to reductions in AKT p-S473 but not p-T308. These GCBC alterations were PTEN-dependent, as they were reversed by PTEN inhibition. Indeed, we found that GCBCs expressed substantially more PTEN than 
did NBCs. This was consequential for PI3K signaling in response to BCR ligation, as GCBCs generated substantially greater amounts of $\operatorname{PtdIns}(4,5) \mathrm{P}_{2}$ than did NBCs. Notably, these differences were at least partly attributable to PTEN as PTEN inhibition in GCBCs increased the generation of $\operatorname{PtdIns}(3,4,5) \mathrm{P}_{3}$ and reduced the generation of $\operatorname{PtdIns}(4,5) \mathrm{P}_{2}$ in response to BCR stimulation. Commensurate with this, PTEN inhibition also increased AKT p-S473 as well as downstream AKT signaling, as measured by p-S6 generation. Finally, we closed the loop by demonstrating that inhibition of AKT enhanced upstream BCR signaling in GCBCs, but did not affect NBCs. A schema for how GCBCs BCR signaling is remodeled via this AKT feedback is shown in Supplementary Fig. 6.

Our finding that PTEN inhibition altered PtdIns $(3,4,5) \mathrm{P}_{3}$ : PtdIns $(4,5) \mathrm{P}_{2}$ ratios after BCR ligation strongly implicates PTEN as a lynchpin controlling differential responses of GCBCs vs. NBCs. Indeed, PTEN expression and/or induction has also been implicated during responses to low or high dose TCR signaling that leads in turn to Treg or T effector generation in vitro ${ }^{40}$. PTEN has also been connected to maintaining B cell anergy ${ }^{41}$. SHIP-1, which generates PtdIns $(3,4,) \mathrm{P}_{2}$ from PtdIns $(3,4,5) \mathrm{P}_{3}$, could also play a role ${ }^{42,43}$; SHIP-1 is hyper-phosphorylated and thereby more likely active in GCBCs compared to $\mathrm{NBCs}^{15}$. In line with our previous finding, we found here that PTEN inhibition also increased PtdIns $\left(3,4, \mathrm{P}_{2}\right.$ upon BCR stimulation, further suggesting that PI3K in GCBCs is functional, but overridden by its key regulatory phosphatases. Finally, there is likely a "forward reaction", to generate PtdIns $(4,5) \mathrm{P}_{2}$ that occurs downstream of BCR ligation in both NBCs and $\mathrm{GCBCs}{ }^{44,45}$.

How does the generation of different amounts of $\operatorname{Ptd} \operatorname{Ins}(3,4,5) \mathrm{P}_{3}$ connect to differences in phosphorylation of AKT? We propose that the different affinities of PH-domains in PDK1 and in Sin 1 (part of the TORC2 complex that is the kinase for AKT-S473) can explain why, under limiting degrees of PI3K-dependent PtdIns $(3,4,5) \mathrm{P}_{3}$ generation, AKT-T308 is preferentially phosphorylated. The affinity of $\mathrm{PDK} 1$ for $\operatorname{Ptd} \operatorname{Ins}(3,4,5) \mathrm{P}_{3}$ is $\sim 1.6 \mathrm{nM}$ while that of $\operatorname{Sin} 1$ for PtdIns $(3,4,5) \mathrm{P}_{3}$ is $\sim 141 \mathrm{nM}^{23,24}$. Thus, PDK1 will activate at substantially lower levels of PtdIns $(3,4,5) \mathrm{P}_{3}$ generation compared to Sin1 (mTORC2) and so T308 phosphorylation will occur selectively upon minimal PI3K activation. Further, in GCBCs, a low concentration of PtdIns $(3,4,5) \mathrm{P}_{3}$ is partly compensated for by increased amounts and activity of PDK1 that in turn can generate AKT p-T308. In this scenario, the amount of PtdIns $(3,4,5) \mathrm{P}_{3}$ generated by BCR ligation in GCBCs is at the lower end of the doseresponse curve for mTORC2 activation. Consistent with this, we found that p-S473 generation was sensitive to PTEN inhibitor-based PtdIns $(3,4,5) \mathrm{P}_{3}$ modulation in GCBCs.

Our findings point to a mechanism of PI3K signal diversification that explains how different analog levels of signaling (i.e. different degrees of PtdIns $(3,4,5) \mathrm{P}_{3}$ generation) are translated into meaningfully different biological outcomes of AKT-mediated signal transduction. We propose that the differential phosphorylation of the two major activating sites on AKT, as observed in GCBCs vs. NBCs, is a key factor in redirecting target specificity. This concept was previously supported in other cell types ${ }^{31}$, albeit not B cells, by a genetic approach that demonstrated that T308 and S473 phosphorylation endowed AKT with different capabilities to phosphorylate distinct substrates. Analogously, in Treg generation and function TCR signal strength is correlated with differential p-T308 vs. p-S473 phosphorylation, with a 
stronger signal needed for generating p-S473. A weaker signal generates Treg and this in turn correlates with different AKT substrate specificity ${ }^{29}$. Treg activation leads to only AKT p-T308, whereas phosphorylation of both T308 and S473 leads to the loss of Treg suppressive function ${ }^{46}$.

We previously showed that BCR signals can induce a transient phosphorylation of AKT at S473, which then allows AKT to mediate efficient FOXO1 phosphorylation ${ }^{16}$. Here, using newly available reagents, we found that GCBCs spontaneously have detectable AKT pT308. These findings help explain our prior finding that dual AKT phosphorylation is required for FOXO1 inactivation ${ }^{31}$, since in GCBCs p-T308 is already present while p-S473 is BCR signal-inducible. It is likely that mTORC1 signals are also regulated differently in GCBC vs NBC. There could be several mechanisms by which mTORC1 signaling is altered in GCBCs. As one example, we found GCBCs have very high amounts of p-AMPK, which is known to inhibit mTORC1 activity (our unpublished observations).

The mechanisms underlying how the balance of phosphorylation at T308 and S473 affects substrate specificity are not yet elucidated: phosphorylation could modulate substrate affinity, enzyme kinetics (thus favoring either high affinity substrates or a more broad distribution), AKT localization, or binding to cofactors. Other factors may also contribute to alteration of phosphorylation states of AKT targets, including differential expression of phosphatases that sculpt the steady state representation of AKT target phosphorylation states $^{47}$.

It was noteworthy that AKT target specificity was so pervasively altered in GCBCs compared to NBCs. The retargeting of kinase activity is a powerful way to coordinately change signaling and responses without changing gene expression of the kinase itself. This novel aspect of the differential signaling mechanisms of GCBCs and NBCs is likely to be used in other cell types and contexts. Hence, our datasets of differentially AKTphosphorylated targets will represent a resource for future exploration, enabling further understanding of PI3K/AKT signal transduction. Though AKT phosphorylates a wide variety of targets ${ }^{48}$, relatively little is known of the details or functional consequences of such events. Strikingly, all the identified negative regulators studied here are novel AKT substrates, in that we could find no published reports of them nor could we find them annotated in the Phosphosite Plus database (www.phosphosite.org) ${ }^{49}$. Thus, we have also added meaningfully to understanding AKT function as well as how activities of CSK, SHP-1 and HPK1 - enzymes that themselves regulate multiple signaling pathways in diverse cell types - are regulated in a signal-dependent fashion.

Our results advance prior studies that have shed light on how BCR signaling is negatively regulated in GCBCs. Initially, we showed that general phosphatase activity was high in GCBCs and provided evidence that SHP-1 and SHIP-1 were in a more phosphorylated and thus more activated state ${ }^{15}$. We also found increased Ser/Thr phosphatase enzymatic activity in GCBCs lysates but did not implicate any specific phosphatase ${ }^{15}$. Here we directly implicate the dual-specificity phosphatase PTEN, which is most well-known for its inositol phosphatase activity but which also dephosphorylates Ser/Thr and Tyr-linked phosphate groups $^{50}$. Others have also investigated GC-specific BCR negative regulation. He et al. ${ }^{51}$ 
found that IgE BCRs in the GC were particularly unfit to transmit needed signals and thus IgE GCBCs were strongly selected against. Haniuda et al. ${ }^{52}$ attributed this to inherent signaling differences in the IgE ectodomain. Zikherman and colleagues used a Nur77-GFP reporter that, while confirming that GCBCs overall have reduced BCR signaling, allowed them to identify a subset of GCBCs with LZ phenotype that showed a higher degree of BCR signals $^{32}$. It will be intriguing to connect our current mechanistic studies to determine how AKT signals may be affected in such Nur77+ GCBCs.

GCBCs join several other B lineage cells in which BCR signal rewiring was demonstrated. Immature B cells have enhanced sensitivity to apoptosis-inducing BCR signaling, which was attributed to enhanced $\mathrm{Ca}^{2+}$ signaling without PKC activation ${ }^{53}$, possibly a consequence of reduced membrane cholesterol in immature B cells ${ }^{54}$. Much like GCBCs, anergic B cells increase phosphatase activity to reduce BCR signal amplitude ${ }^{55}$. Interestingly, upregulation of PTEN has also been implicated in anergy induction, along with higher SHP-1 and SHIP-1 activity $^{55}$. Infection and malignant transformation can also remodel proximal BCR signaling. Uncoupling of p-SYK generation from PLC- $\gamma$ phosphorylation was a hallmark of CLL and correlated with clinical status ${ }^{56}$. Together these studies reveal several mechanisms and contexts for rewiring BCR signal transduction.

Negative feedback loops are typically mediated within the same protein or by interaction with a protein that is adjacent in the pathway. A relevant example includes BCR signal dependent phosphorylation of SHP-1 that in turn activates SHP-1 to dephosphorylate SYK/LYN and complete the feedback ${ }^{1535,57}$. The AKT-dependent GCBCs circuit delineated here is unusual in that AKT targets and activates not one but several negative regulators of BCR signaling that are topologically upstream of AKT itself.

In total, these studies define mechanistically how BCR signaling is rewired in GCBCs and, in addition, report novel functions and substrates of AKT. Both of these observations will also serve as models for further investigation in analogous systems and cell types as it is likely that these paradigms will operate more widely beyond BCR signaling in GCBCs.

\section{Methods}

\section{Mice and Immunization}

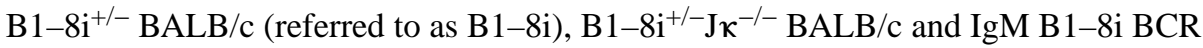
transgenic $\mathrm{BALB} / \mathrm{c}$ mice (referred to as MEG) were previously described ${ }^{15,58,59}$. Mice of either strain were used as sources of GCBCs and NBCs, as indicated in figure legends. Mice were maintained under specific-pathogen-free conditions supervised by the University of Pittsburgh Institutional Animal Care and Use Committee (IACUC). 6-to-12 weeks old, ageand sex-matched mice were immunized i.p. with $50 \mu \mathrm{g}$ NP-CGG precipitated in Alum. Mice were analyzed at day 13 to 14 post immunization, or as otherwise indicated in the figure legends.

\section{Cell Preparation and Treatment}

NBCs were purified from unimmunized mice and GCBCs were purified from day 13 to 14 NP-CGG immunized mice. The cell purification process was performed as previously 
described $^{16}$. Briefly, splenic NBCs were purified by negative selection using a biotinconjugated antibody cocktail (antibodies against CD43, CD4, CD8, CD11b, CD11c, Gr-1 and CD138), followed by magnetic bead-depletion of labeled cells (B cell purity $\geq 95 \%$ ). For GCBC purification from B1-8i mice, biotin-conjugated anti-IgD and anti-CD38 antibodies were added to the $\mathrm{B}$ cell purification cocktail; for GCBC purification from MEG mice, biotin-conjugated anti-CD38 antibody was added to the B cell purification cocktail (GCBC purity 290\%). Purified cells or total splenocytes were warmed to $37{ }^{\circ} \mathrm{C}$ with $5 \%$ $\mathrm{CO}_{2}$ in B cell medium (RPMI-1640 medium supplemented with $10 \% \mathrm{FBS}$, penicillin/ streptomycin, glutamine and $50 \mu \mathrm{M} \beta$-mercaptoethanol) for 30 to $40 \mathrm{~min}$ and stimulated with $20 \mu \mathrm{g} / \mathrm{ml}$ goat anti-mouse IgM ( $\mu$-chain specific, Jackson ImmunoResearch) or 200 $\mathrm{ng} / \mathrm{ml}$ NP-Ficoll (LGC Biosearch Technologies) as indicated in the figure legends. Endotoxin was removed from antibodies used for stimulation with ToxinEraserTM Endotoxin Removal Kit (GenScript) and endotoxin levels were tested with a LAL assay kit (GenScript) (endotoxin $<0.5 \mathrm{EU} / \mathrm{ml}$ ). For experiments using inhibitors, cells were treated with $10 \mu \mathrm{M}$ AKT1/2 kinase inhibitor (Calbiochem) or $10 \mu \mathrm{M}$ PTEN inhibitor (SF1670, Calbiochem) dissolved in DMSO or the same amount of DMSO alone, as described in figure legends, prior to stimulation. For experiments measuring basal amounts of protein expression or phosphorylation, purified cells were analyzed immediately after purification.

For AKT phospho-substrate antibody immunoblotting, the different cell populations were sorted by FACSAria (BD Immunocytometry Systems). The gating strategy of sorting and post-sort testing is shown in Supplementary Fig. 1b. Briefly, NBCs were identified as $\mathrm{CD} 19^{+} \mathrm{CD} 93^{-}$live singlets; in vivo and in vitro activated $\mathrm{B}$ cells as $\mathrm{CD} 19^{+} \mathrm{CD} 86^{+}$live singlets with increased forward scatter; $\mathrm{GCBCs}$ were defined as $\mathrm{CD} 19^{+} \mathrm{CD} 95^{+} \mathrm{CD} 38^{-}$live singlets. Purification led in general to over $98 \%$ purity of target cell populations. To generate in vitro activated B cells, magnetic bead-purified NBCs (as described above) were cultured in RPMI 1640 media in the presence of $5 \mu \mathrm{g} / \mathrm{ml} \mathrm{CpG} \mathrm{ODN} 1826$ (Invivogen) for $48 \mathrm{~h}$ at $37^{\circ} \mathrm{C}$. To generate in vivo activated B cells, B1-8i ${ }^{+/-} \mathrm{JK}^{-/-}$mice were given $50 \mu \mathrm{g}$ NP-Ficoll (LGC Biosearch Technologies) i.p., and cells were prepared $48 \mathrm{~h}$ later.

\section{Immunoblot Analysis}

Whole cell lysates were prepared by direct lysing and boiling samples in Laemmli buffer supplemented with $\beta$-mercaptoethanol. For the experiment in Supplementary Fig. 2, the same numbers of GCBCs and NBCs were lysed in RIPA buffer supplemented with protease and phosphatase inhibitor cocktail (ThermoFisher Scientific). Protein concentrations were measured with a BCA Protein Assay Kit (Pierce ${ }^{\mathrm{TM}}$ ) and equal amount of protein were loaded in SDS-PAGE gels for immunoblot. The samples were then blotted with the following antibodies against: Actin (clone: C4/actin, BD Biosciences), total-ERK1/2 (clone: 137F5, Cell Signaling Technology), phospho-SYK (Y352; rabbit polyclonal, Cell Signaling Technology), phospho-AKT (T308; clone: D25E6, Cell Signaling Technology), phosphoAKT (S473; clone: D9E, Cell Signaling Technology ), phospho-PDK1 (S241; rabbit polyclonal, Cell Signaling Technology), total-PDK1 (clone: D37A7, Cell Signaling Technology), PTEN (clone: D4.3, Cell Signaling Technology), phospho-LYN (Y396; clone: EP503Y, Abcam), phospho-LYN (Y507; rabbit polyclonal, Cell Signaling Technology), AKT phospho-substrate motif (clone: 110B7E, Cell Signaling Technology), PI(3) Kinase 
p1108 (clone: D1Q7R, Cell Signaling Technology), PI(3) Kinase p110a (clone: C73F8, Cell Signaling Technology), S6 Ribosomal Protein (clone: 5G10, Cell Signaling Technology), CSK (clone: C74C1, Cell Signaling Technology), CD45 (clone: D4H7K, Cell Signaling Technology), Phospho-Src Family (Tyr416; clone: D49G4, Cell Signaling Technology), Phospho-Src (Tyr527; Rabbit polyclonal, Cell Signaling Technology), total Src (Rabbit polyclonal, Cell Signaling Technology), total SYK (clone: D3Z1E, Cell Signaling Technology), AKT (pan) (clone; C67E7, Cell Signaling Technology), HPK1 (Rabbit polyclonal, Cell Signaling Technology), LYN (clone: C13F9, Cell Signaling Technology), SHP-1 (clone: C14H6, Cell Signaling Technology), ARP2 (clone:D85D5, Cell Signaling Technology) and Phospho-Threonine (clone:D85D5, Cell Signaling Technology). Densitometry was performed using ImageJ software ${ }^{60}$. Immunoblots in Supplementary Fig. 2 were loaded with an equal amount of protein per lane. Actin was used as a normalization loading control for other immunoblots.

\section{Flow Cytometry}

Cells were fixed and permeabilized in saponin-based Perm/Wash buffer (Cat\#: 554732, BD Biosciences) supplemented with $1.5 \%$ paraformaldehyde (PFA) for $10 \mathrm{~min}$ at $20-25{ }^{\circ} \mathrm{C}$ followed by $30 \mathrm{~min}$ on ice. For experiments focused on detecting basal level of phosphorylation, cells were fixed by directly disrupting spleens into RPMI containing $1.5 \%$ PFA and incubating for $15 \mathrm{~min}$ at $20-25^{\circ} \mathrm{C}$ ("instant fix") before permeabilization with Perm/Wash buffer. Fc receptors on cells were blocked by incubating with anti-CD16/CD32 antibody (clone: $2.4 \mathrm{G} 2$, prepared in the lab) prior to staining with fluorochrome-conjugated antibodies against: phospho-S6 (S235/236; clone: D57.2.2E, Cell Signaling Technology), phospho-AKT (S473; clone: M89-61, BD Biosciences), phospho-Btk (Y223/Itk pY180; clone: N35-86, BD Biosciences), phospho-PLC- $\gamma 2$ (Y759; clone: K86-689.37, BD Biosciences) CXCR4 (clone: L276F12, BioLegend), CD86 (clone: GL1, prepared and conjugated in our lab), B220 (clone: RA3-6B2, BD Bioscience and BioLegend), PNA (Vector Lab, conjugated in our lab), CD95 (clone: Jo2, BD Biosciences), IgM (goat polyclonal, Jackson ImmunoResearch), Ig $\lambda$ (polyclonal, Southern Biotech, conjugated in our lab). For phospho-AKT (T308), cells were stained with unconjugated rabbit anti-mouse phospho-AKT (T308; clone: D25E6, Cell Signaling Technology), followed by secondary Cy3- or Alexa647-conjugated anti-rabbit secondary antibody (ThermoFisher Scientific).

Stained cells were analyzed on a BD LSRII or Fortessa flow cytometer. Data were analyzed with FlowJo 10 software. The following gating strategy was used for cells from B1-8i or MEG mice: GCBCs were gated as B220 $\lambda^{+} \mathrm{PNA}^{+}\left(\right.$or $\left.\mathrm{CD}^{+} 5^{+}\right)$cells, non-GCBCs were gated as $\mathrm{B} 220^{+} \lambda{ }^{+} \mathrm{PNA}^{-}\left(\right.$or $\mathrm{CD}^{-} 5^{-}$) cells and $\mathrm{NBCs}$ were gated as $\mathrm{B} 220^{+} \lambda \lambda^{-} \mathrm{PNA}^{-}$(or $\mathrm{CD}^{-} 5^{-}$) cells (Supplementary Fig. 1a).

\section{Measurement of phosphatidylinositol abundance}

$1 \times 10^{6} \mathrm{~B}$ cells were collected per time point. Samples were processed per the manufacturers' instructions included in the Echelon Biosciences Mass ELISA kits for PtdIns(4,5) $\mathrm{P}_{2}(\mathrm{~K}-4500)$, PtdIns $(3,4,5) \mathrm{P}_{3}$ (K-2500s) and PtdIns(3,4) $\mathrm{P}_{2}$ (K-3800). The mass ELISA assays were measured at $450 \mathrm{nM}$ on a Molecular Devices SpectraMax i3 plate reader. The standard curve was fit assuming a sigmoidal dose-response with variable slope 
and the amount of phosphatidylinositol in each sample was extrapolated in the GraphPad Prism 7 software package.

\section{Immunoprecipitation of AKT substrates and Mass Spectrometric Analysis}

$2 \times 10^{6} \mathrm{~B}$ cells were collected and lysed with a buffer containing $25 \mathrm{mM}$ Tris-HCl pH 7.4, $150 \mathrm{mM} \mathrm{NaCl}, 1 \mathrm{mM}$ EDTA, $1 \%$ NP40, 5\% glycerol that was supplemented with Roche Complete $\mathrm{C}$ protease and PhosStop phosphatase inhibitors. Lysates were sonicated and centrifuged to remove insoluble debris and the remaining lysate was incubated with magnetic bead-conjugated AKT phospho-substrate antibody (Clone: 110B7E, Cell signaling Technologies, Cat \#: 8050) for $12 \mathrm{~h}$ at $4{ }^{\circ} \mathrm{C}$. The beads were washed twice with lysis buffer and proteins were eluted from the beads with $8 \mathrm{M}$ urea (Sigma, U5128) and $0.1 \mathrm{M}$ Tris-HCl at $\mathrm{pH}$ 8.5. The filter aided sample preparation method (FASP) was used to generate tryptic peptides and desalted using $\mathrm{C} 18$ spin columns ${ }^{61}$. Peptides were suspended in $0.1 \%$ formic acid and resolved with liquid chromatography tandem mass spectrometry (LC-MS/MS) using a system comprised of a Waters nanoAcuity HPLC in-line with either a LTQ/ OrbitrapVelos Elite hybrid mass spectrometer (Thermo-Fisher) or a Q Exactive mass spectrometer (Thermo-Fisher). Solvent A ( $0.1 \%$ formic acid in HPLC-grade water) and solvent B ( $0.1 \%$ formic acid in $100 \%$ acetonitrile) were used as the mobile phase. Peptides were then eluted onto a capillary column $(75 \mu \mathrm{m}$ inner diameter $\times 360 \mu \mathrm{m}$ outer diameter $\times$ $15 \mathrm{~cm}$ long; Polymicro Technologies) $5 \mu \mathrm{m}$ particle size, 125 pore size $\mathrm{C}-18$ silica-bonded stationary phase (Phenomenex) and resolved using a 100 min gradient at the flow rate of 0.2 $\mu \mathrm{l} / \mathrm{min}$ (3-33\% B for $90 \mathrm{~min}, 33-80 \%$ B for $2 \mathrm{~min}$, constant at $80 \%$ B for $6 \mathrm{~min}$, and then $80-0 \%$ B for $2 \mathrm{~min}$ ). Data were collected in positive ionization mode. PEAKS8 software was used to sequence and identify peptides in each sample using a decoy search at a $1 \%$ false discovery rate using the UniProt murine database. Label free quantitation was performed using the quantitative module in the PEAKS8 software.

\section{Bioinformatics}

The following criteria was used to identify AKT substrates enriched in different cell types: 1) peptide(s) corresponding to a protein had to be identified in a sample type in the MS analysis and 2) if a given substrate was identified in multiple sample groups, its abundance had to be more than three-fold greater based on the label free quantitation of the mass spectrometric data to be assigned as specific to a group(s). The PANTHER pathway analysis software was used to perform a statistical overrepresentation test to identify biological pathways that were differentially targeted by AKT in the NBCs and $\mathrm{GCBCs}^{62}$.

\section{AKT co-immunoprecipitation Analysis}

NBCs or GCBCs were activated with anti-IgM antibody in the presence or absence of $10 \mu \mathrm{M}$ AKT1/2 kinase inhibitor (Calbiochem). Cells were lysed in a buffer containing $25 \mathrm{mM}$ Tris$\mathrm{HCl} \mathrm{pH} \mathrm{7.4,} 150 \mathrm{mM} \mathrm{NaCl}, 1 \mathrm{mM}$ EDTA, 1\% NP40, 5\% glycerol that was supplemented with Roche Complete $\mathrm{C}$ protease and Roche PhosStop phosphatase inhibitors and incubated with agarose beads coated with magnetic bead-conjugated AKT phospho-substrate antibody (Clone: 110B7E, Cell signaling Technologies, Cat \#: 8050) for $2 \mathrm{~h}$ at $4{ }^{\circ} \mathrm{C}$. Immunoblot was then used to determine if specific proteins were immunoprecipitated by the antibody. The following antibodies used in immunoblot were all purchased from Cell Signaling 
Technology: antibodies against CSK (clone: C74C1), SHP-1 (clone: C14H6), HPK1 (rabbit polyclonal), ARP2 (clone: D85D5) and LYN (rabbit polyclonal).

\section{In vitro AKT Enzyme Reactions}

Recombinant preactivated AKT (1 $\mu \mathrm{g}$, Sigma SRP5001) was reacted with $1 \mathrm{mM}$ ATP (Sigma) and $1 \mu \mathrm{g}$ of recombinant CSK (abcam), SHP-1 (abcam), LYN (abcam) or HPK1 (abcam) for $30 \mathrm{~min}$ at $37^{\circ} \mathrm{C}$ in an AKT reaction buffer containing $25 \mathrm{mM}$ MOPS, $\mathrm{pH} 7.2$, $12.5 \mathrm{mM}$ glycerol 2-phosphate, $25 \mathrm{mM} \mathrm{MgCl}_{2}, 5 \mathrm{mM}$ EGTA,2 mM EDTA and $0.25 \mathrm{mM}$ DTT. Reactions were quenched with Laemmli buffer and resolved by SDS-PAGE. immunoblot was performed using the AKT phospho-substrate antibody to detect AKT mediated phosphorylation of the target proteins. To determine the phosphorylation status of the purchased recombinant pre-activated AKT, we performed an immunoprecipitation with anti-AKT p-S473 followed by immunoblot of the immunoprecipitated and nonimmunoprecipitated fractions, along with the input material, using both anti-AKT p-S473 and anti-AKT p-T308, which revealed that the material was a mixture of single and doubly phosphorylated enzyme (data not shown).

Mass spectrometry was used to identify the AKT phosphorylation sites on the substrate as follows: In vitro kinase reactions were performed as detailed above and quenched with a buffer containing $8 \mathrm{M}$ urea and $0.1 \mathrm{M}$ Tris- $\mathrm{HCl}$ at $\mathrm{pH}$ 8.5. FASP was used to prepare tryptic peptides that were analyzed on a LTQ/Orbitrap Velos Elite hybrid mass spectrometer. PEAKS8 software was used to sequence and identify peptides in each sample using a decoy search at a $1 \%$ false discovery rate and phospho-serine and phospho-threonine were set as variable modifications.

\section{Enzyme Assays}

CSK enzyme assays: $1 \mu \mathrm{g}$ of recombinant CSK (abcam) was incubated with $1 \mathrm{mM}$ of ATP in the presence or absence (as negative control) of $1 \mu \mathrm{g}$ of AKT (Sigma) in AKT reaction buffer at $37{ }^{\circ} \mathrm{C}$ for $30 \mathrm{~min}$. After dialysis to remove excess ATP and ADP, $0.1 \mu \mathrm{g}$ equivalent of CSK from the AKT kinase reaction or the negative control reaction was reacted with $1 \mu \mathrm{g}$ of recombinant LYN (abcam) and $1 \mathrm{mM}$ ATP. Immunoblotting was performed to detect phospho-LYN (Y507) (rabbit polyclonal, Cell Signaling Technology) and total LYN (clone: C13F9, Cell Signaling Technology).

SHP-1 enzyme assays: $1 \mu \mathrm{g}$ of recombinant SHP-1 (abcam) was incubated with $1 \mathrm{mM}$ of ATP in the presence or absence (as negative control) of $1 \mu \mathrm{g}$ of AKT (Sigma) in AKT reaction buffer at $37^{\circ} \mathrm{C}$ for $30 \mathrm{~min}$. The reactions were dialyzed to remove unreacted ATP and ADP. $0.1 \mu \mathrm{g}$ of SHP-1 obtained from the reaction with AKT or the negative control reaction was reacted with $50 \mathrm{mM}$ of p-nitrophenyl phosphate (PNPP) in $25 \mathrm{mM}$ HEPES, pH 7.2, $50 \mathrm{mM} \mathrm{NaCl}, 2.5 \mathrm{mM}$ EDTA, $5 \mathrm{mM}$ DTT. The absorbance at $405 \mathrm{~nm}$ was monitored on a Molecular Devices SpectraMax i3 microplate reader.

HPK1 enzyme assays: $1 \mu \mathrm{g}$ of recombinant HPK1 (abcam) was incubated with $1 \mathrm{mM}$ of ATP in the presence or absence (as negative control) of $1 \mu \mathrm{g}$ of AKT (Sigma) in AKT reaction buffer at $37{ }^{\circ} \mathrm{C}$ for $30 \mathrm{~min}$. After dialysis to remove excess ATP and ADP, the HPK1 reactions were incubated with $1 \mu \mathrm{g}$ recombinant BLNK. Immunoblot using an 
antibody that recognized phospho-theronine residues was used to monitor BLNK phosphorylation by HPK1 (rabbit polyclonal, Cell Signaling Technology) and total BLNK protein (clone: D3P2H, Cell Signaling Technology).

\section{Quantification and Statistical Analysis}

Statistical analysis was performed with Prism software (GraphPad Software). For comparing two groups, $P$-values were determined using Student's $t$-tests (two-tailed). For comparing more than two groups, One-Way ANOVA followed by Tukey test was applied. Two-Way ANOVA followed by Sidak's multiple comparisons test was used for evaluating data with two factors. Differences between groups were considered significant for $P$-values $<0.05$.

\section{Data availability Statement}

The data that support the findings of this study are available from the corresponding author upon reasonable request. Reagents and methods used in this paper are described in the Life Sciences Reporting Summary, available online.

\section{Supplementary Material}

Refer to Web version on PubMed Central for supplementary material.

\section{Acknowledgements}

We thank Drs. L. Garrett-Sinha, L. Kane, G. Delgoffe and B. Su for critical reading of the manuscript. We thank Dr. B. Elsner for useful discussions. We thank S. Joachim for supporting experimental procedures. Supported by NIH grants R01 AI105018 to MJS

\section{References}

1. De Silva NS \& Klein U Dynamics of B cells in germinal centres. Nat Rev Immunol 15, 137-148 (2015). [PubMed: 25656706]

2. Shlomchik MJ \& Weisel F Germinal center selection and the development of memory B and plasma cells. Immunol Rev 247, 52-63 (2012). [PubMed: 22500831]

3. Mesin L, Ersching J \& Victora GD Germinal Center B Cell Dynamics. Immunity 45, 471-482 (2016). [PubMed: 27653600]

4. Weisel FJ, Zuccarino-Catania GV, Chikina M \& Shlomchik MJ A Temporal Switch in the Germinal Center Determines Differential Output of Memory B and Plasma Cells. Immunity 44, 116-130 (2016). [PubMed: 26795247]

5. Basso K \& Dalla-Favera R BCL6: master regulator of the germinal center reaction and key oncogene in B cell lymphomagenesis. Adv Immunol 105, 193-210 (2010). [PubMed: 20510734]

6. Bunting KL et al. Multi-tiered Reorganization of the Genome during B Cell Affinity Maturation Anchored by a Germinal Center-Specific Locus Control Region. Immunity 45, 497-512 (2016). [PubMed: 27637145]

7. Good-Jacobson KL et al. Regulation of germinal center responses and B-cell memory by the chromatin modifier MOZ. Proc Natl Acad Sci U S A 111, 9585-9590 (2014). [PubMed: 24979783]

8. Liu D et al. T-B-cell entanglement and ICOSL-driven feed-forward regulation of germinal centre reaction. Nature 517, 214-218 (2015). [PubMed: 25317561]

9. Lu P, Shih C \& Qi H Ephrin B1-mediated repulsion and signaling control germinal center T cell territoriality and function. Science 356 (2017).

10. Zaretsky I et al. ICAMs support B cell interactions with T follicular helper cells and promote clonal selection. J Exp Med 214, 3435-3448 (2017). [PubMed: 28939548] 
11. Wang Y et al. Germinal-center development of memory B cells driven by IL-9 from follicular helper T cells. Nat Immunol 18, 921-930 (2017). [PubMed: 28650481]

12. Yan $\mathrm{H}$ et al. Plexin B2 and Semaphorin $4 \mathrm{C}$ Guide $\mathrm{T}$ Cell Recruitment and Function in the Germinal Center. Cell reports 19, 995-1007 (2017). [PubMed: 28467912]

13. Kräutler NJ et al. Differentiation of germinal center B cells into plasma cells is initiated by highaffinity antigen and completed by Tfh cells. J Exp Med (2017).

14. Harwood NE \& Batista FD New insights into the early molecular events underlying B cell activation. Immunity 28, 609-619 (2008). [PubMed: 18482567]

15. Khalil AM, Cambier JC \& Shlomchik MJ B Cell Receptor Signal Transduction in the GC Is ShortCircuited by High Phosphatase Activity. Science 336, 1178-1181 (2012). [PubMed: 22555432]

16. Luo W, Weisel F \& Shlomchik MJ B Cell Receptor and CD40 Signaling Are Rewired for Synergistic Induction of the c-Myc Transcription Factor in Germinal Center B Cells. Immunity 48, 313-+ (2018). [PubMed: 29396161]

17. Sander S et al. PI3 Kinase and FOXO1 Transcription Factor Activity Differentially Control B Cells in the Germinal Center Light and Dark Zones. Immunity 43, 1075-1086 (2015). [PubMed: 26620760]

18. Dominguez-Sola D et al. The FOXO1 Transcription Factor Instructs the Germinal Center Dark Zone Program. Immunity 43, 1064-1074 (2015). [PubMed: 26620759]

19. Calado DP et al. The cell-cycle regulator c-Myc is essential for the formation and maintenance of germinal centers. Nat Immunol 13, 1092-1100 (2012). [PubMed: 23001146]

20. Dominguez-Sola D et al. The proto-oncogene MYC is required for selection in the germinal center and cyclic reentry. Nat Immunol 13, 1083-1091 (2012). [PubMed: 23001145]

21. Ersching J et al. Germinal Center Selection and Affinity Maturation Require Dynamic Regulation of mTORC1 Kinase. Immunity 46, 1045-1058 e1046 (2017). [PubMed: 28636954]

22. Toker A \& Newton AC Cellular signaling: pivoting around PDK-1. Cell 103, 185-188 (2000). [PubMed: 11057891]

23. Gokhale NA, Zaremba A, Janoshazi AK, Weaver JD \& Shears SB PPIP5K1 modulates ligand competition between diphosphoinositol polyphosphates and PtdIns(3,4,5)P-3 for polyphosphoinositide-binding domains. Biochemical Journal 453, 413-426 (2013). [PubMed: 23682967]

24. Currie RA et al. Role of phosphatidylinositol 3,4,5-trisphosphate in regulating the activity and localization of 3-phosphoinositide-dependent protein kinase-1. Biochemical Journal 337, 575-583 (1999). [PubMed: 9895304]

25. Liu P et al. PtdIns(3,4,5)P3-Dependent Activation of the mTORC2 Kinase Complex. Cancer Discov 5, 1194-1209 (2015). [PubMed: 26293922]

26. Gan X, Wang J, Su B \& Wu D Evidence for direct activation of mTORC2 kinase activity by phosphatidylinositol 3,4,5-trisphosphate. J Biol Chem 286, 10998-11002 (2011). [PubMed: 21310961]

27. Ramadani $\mathrm{F}$ et al. The PI3K isoforms p110alpha and p110delta are essential for pre-B cell receptor signaling and B cell development. Sci Signal 3, ra60 (2010). [PubMed: 20699475]

28. Sun $\mathrm{H}$ et al. PTEN modulates cell cycle progression and cell survival by regulating phosphatidylinositol 3,4,5,-trisphosphate and Akt/protein kinase B signaling pathway. Proc Natl Acad Sci U S A 96, 6199-6204 (1999). [PubMed: 10339565]

29. Hawse WF, Boggess WC \& Morel PA TCR Signal Strength Regulates Akt Substrate Specificity To Induce Alternate Murine Th and T Regulatory Cell Differentiation Programs. J Immunol 199, 589-597 (2017). [PubMed: 28600288]

30. Yung HW, Charnock-Jones DS \& Burton GJ Regulation of AKT Phosphorylation at Ser473 and Thr308 by Endoplasmic Reticulum Stress Modulates Substrate Specificity in a Severity Dependent Manner. PLoS One 6 (2011).

31. Jacinto E et al. SIN1/MIP1 maintains rictor-mTOR complex integrity and regulates Akt phosphorylation and substrate specificity. Cell 127, 125-137 (2006). [PubMed: 16962653]

32. Mueller J, Matloubian M \& Zikherman J Cutting edge: An in vivo reporter reveals active B cell receptor signaling in the germinal center. J Immunol 194, 2993-2997 (2015). [PubMed: 25725108] 
33. Nowosad CR, Spillane KM \& Tolar P Germinal center B cells recognize antigen through a specialized immune synapse architecture. Nat Immunol 17, 870-877 (2016). [PubMed: 27183103]

34. Ingley E Src family kinases: Regulation of their activities, levels and identification of new pathways. Bba-Proteins Proteom 1784, 56-65 (2008).

35. Zhang JY, Somani AK \& Siminovitch KA Roles of the SHP-1 tyrosine phosphatase in the negative regulation of cell signalling. Semin Immunol 12, 361-378 (2000). [PubMed: 10995583]

36. Wang XH et al. Down-regulation of B Cell Receptor Signaling by Hematopoietic Progenitor Kinase 1 (HPK1)-mediated Phosphorylation and Ubiquitination of Activated B Cell Linker Protein (BLNK). Journal of Biological Chemistry 287, 11037-11048 (2012). [PubMed: 22334673]

37. Higgs HN \& Pollard TD Regulation of actin polymerization by Arp2/3 complex and WASp/Scar proteins. Journal of Biological Chemistry 274, 32531-32534 (1999). [PubMed: 10551802]

38. Levinson NM, Seeliger MA, Cole PA \& Kuriyan J Structural basis for the recognition of c-Src by its inactivator Csk. Cell 134, 124-134 (2008). [PubMed: 18614016]

39. Niiro H \& Clark EA Regulation of B-cell fate by antigen-receptor signals. Nat Rev Immunol 2, 945-956 (2002). [PubMed: 12461567]

40. Hawse WF et al. Cutting Edge: Differential Regulation of PTEN by TCR, Akt, and FoxO1 Controls CD4+ T Cell Fate Decisions. J Immunol 194, 4615-4619 (2015). [PubMed: 25855357]

41. Getahun A, Beavers NA, Larson SR, Shlomchik MJ \& Cambier JC Continuous inhibitory signaling by both SHP-1 and SHIP-1 pathways is required to maintain unresponsiveness of anergic B cells. J Exp Med 213, 751-769 (2016). [PubMed: 27114609]

42. O'Neill SK et al. Monophosphorylation of CD79a and CD79b ITAM motifs initiates a SHIP-1 phosphatase-mediated inhibitory signaling cascade required for B cell anergy. Immunity 35, 746756 (2011). [PubMed: 22078222]

43. Akerlund J, Getahun A \& Cambier JC B cell expression of the SH2-containing inositol 5 phosphatase (SHIP-1) is required to establish anergy to high affinity, proteinacious autoantigens. J Autoimmun 62, 45-54 (2015). [PubMed: 26152931]

44. Xu C et al. A PIP2-derived amplification loop fuels the sustained initiation of B cell activation. Sci Immunol 2 (2017).

45. Saito K et al. BTK regulates PtdIns-4,5-P2 synthesis: importance for calcium signaling and PI3K activity. Immunity 19, 669-678 (2003). [PubMed: 14614854]

46. Crellin NK, Garcia RV \& Levings MK Altered activation of AKT is required for the suppressive function of human CD4(+) CD25(+) T regulatory cells. Blood 109, 2014-2022 (2007). [PubMed: 17062729]

47. Polikowsky HG, Wogsland CE, Diggins KE, Huse K \& Irish JM Cutting Edge: Redox Signaling Hypersensitivity Distinguishes Human Germinal Center B Cells. The Journal of Immunology (2015).

48. Manning BD \& Cantley LC AKT/PKB signaling: navigating downstream. Cell 129, 1261-1274 (2007). [PubMed: 17604717]

49. Hornbeck PV et al. PhosphoSitePlus, 2014: mutations, PTMs and recalibrations. Nucleic Acids Res 43, D512-520 (2015). [PubMed: 25514926]

50. Myers MP et al. P-TEN, the tumor suppressor from human chromosome 10q23, is a dualspecificity phosphatase. Proceedings of the National Academy of Sciences 94, 9052-9057 (1997).

51 . He JS et al. The distinctive germinal center phase of $\operatorname{IgE}(+)$ B lymphocytes limits their contribution to the classical memory response. Journal of Experimental Medicine 210, 2755-2771 (2013). [PubMed: 24218137]

52. Haniuda K, Fukao S, Kodama T, Hasegawa H \& Kitamura D Autonomous membrane IgE signaling prevents IgE-memory formation. Nat Immunol 17, 1109-1117 (2016). [PubMed: 27428827]

53. King LB, Norvell A \& Monroe JG Antigen receptor-induced signal transduction imbalances associated with the negative selection of immature B cells. J Immunol 162, 2655-2662 (1999). [PubMed: 10072508]

54. Karnell FG, Brezski RJ, King LB, Silverman MA \& Monroe JG Membrane Cholesterol Content Accounts for Developmental Differences in Surface B Cell Receptor Compartmentalization and Signaling. Journal of Biological Chemistry 280, 25621-25628 (2005). [PubMed: 15878848] 
55. Franks SE \& Cambier JC Putting on the Brakes: Regulatory Kinases and Phosphatases Maintaining B Cell Anergy. Frontiers in immunology 9, 665 (2018). [PubMed: 29681901]

56. Palomba ML et al. Multidimensional Single-Cell Analysis of BCR Signaling Reveals Proximal Activation Defect As a Hallmark of Chronic Lymphocytic Leukemia B Cells. PLoS One 9, 12 (2014).

57. Zhang Z, Shen K, Lu W \& Cole PA The role of C-terminal tyrosine phosphorylation in the regulation of SHP-1 explored via expressed protein ligation. J Biol Chem 278, 4668-4674 (2003). [PubMed: 12468540]

58. Hannum LG, Haberman AM, Anderson SM \& Shlomchik MJ Germinal center initiation, variable gene region hypermutation, and mutant B cell selection without detectable immune complexes on follicular dendritic cells. J Exp Med 192, 931-942 (2000). [PubMed: 11015435]

59. Sonoda E et al. B cell development under the condition of allelic inclusion. Immunity 6, 225-233 (1997). [PubMed: 9075923]

60. Schneider CA, Rasband WS \& Eliceiri KW NIH Image to ImageJ: 25 years of image analysis. Nat Methods 9, 671-675 (2012). [PubMed: 22930834]

61. Wisniewski JR, Zougman A, Nagaraj N \& Mann M Universal sample preparation method for proteome analysis. Nat Methods 6, 359-362 (2009). [PubMed: 19377485]

62. Mi H, Muruganujan A, Casagrande JT \& Thomas PD Large-scale gene function analysis with the PANTHER classification system. Nat Protoc 8, 1551-1566 (2013). [PubMed: 23868073] 


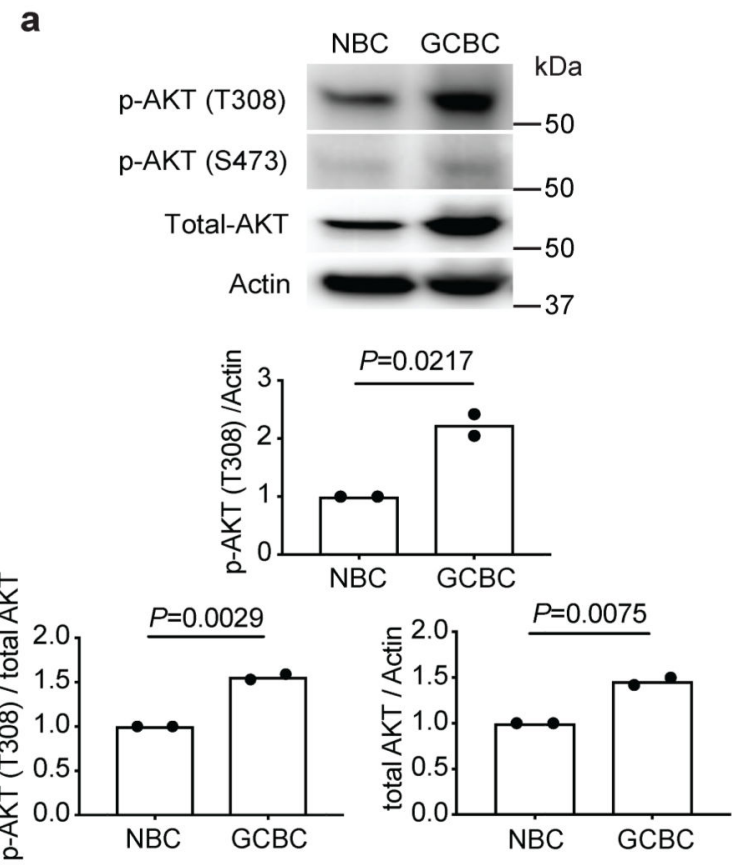

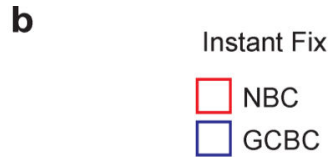
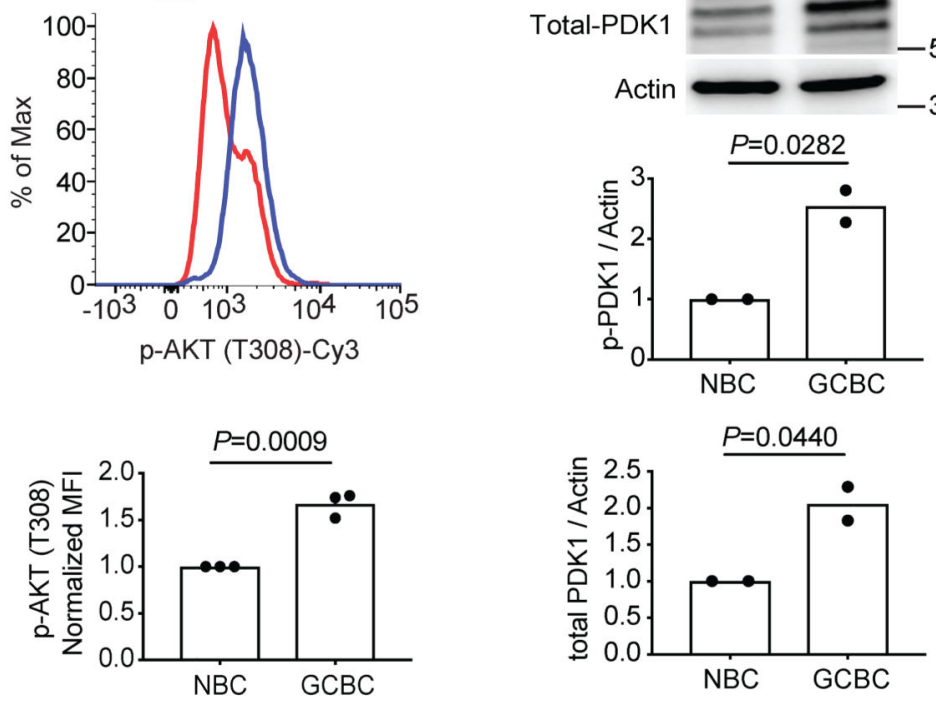

d

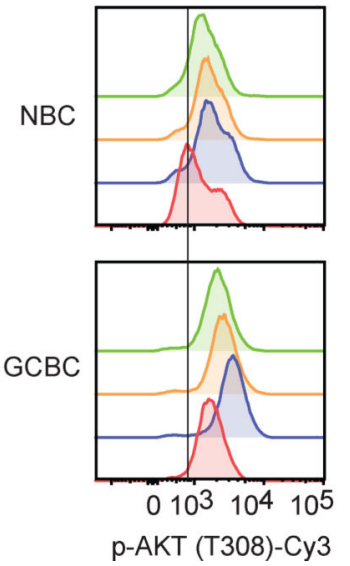

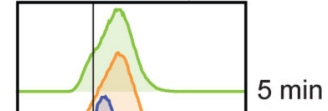

$3 \mathrm{~min}$

Fig.1 |. Phosphorylation of AKT is altered in GCBCs compared to NBCs.

a, AKT protein expression and phosphorylation was analyzed by immunoblot with freshly purified B1-8i NBCs and GCBCs (n=2); lower graph shows the quantitation of bands, with bars showing means of replicates. The ratios were normalized to those of NBCs, given a value of 1. b, Splenocytes from immunized MEG mice $(n=3)$ were instantly fixed in $1.5 \%$ PFA and then analyzed by flow cytometry; lower graph shows fold change of median fluorescence intensity (MFI) and was normalized to NBCs as 1. Graph shows the quantitation as in (b). c, Western blot for p-PDK1(S241) and total PKD1 in purified MEG NBCs and GCBCs $(n=2)$; lower graphs show the quantitation of bands as in (b). The ratios were normalized to those of NBCs, given a value of 1. d, Total splenocytes from immunized MEG mice $(n=3)$ were stimulated with anti-IgM for indicated time points and $\mathrm{p}$-AKT in 
GCBCs and NBCs was analyzed by flow cytometry. e, Purified MEG NBCs and GCBCs $(n=2)$ were rested for 40 minutes and stimulated with anti-IgM for indicated time points and cell lysates were analyzed by immunoblot. Data represent two independent experiments with cells pooled from two mice per group in each experiment $(\mathbf{a}, \mathbf{c}, \mathbf{e})$ or three independent experiments with one mouse tested in each experiment $(\mathbf{b}, \mathbf{d})$. $P$ values, two-tailed unpaired Student's $t$-test (a,b,c). (p-AKT(T308)/Actin: $\mathrm{t}=6.676$, d.f.=2; p-AKT(T308)/total AKT: $\mathrm{t}=18.67$, d.f. $=2$; total AKT/Actin: $\mathrm{t}=11.50, \mathrm{df}=2$; $\mathrm{p}-\mathrm{AKT}(\mathrm{T} 308)$ normalized MFI: $\mathrm{t}=8.758$, d.f.=4; p-PDK1/Actin: $t=5.830, \mathrm{df}=2$; total PDK1/Actin: $\mathrm{t}=4.609, \mathrm{df}=2$ ). 
a
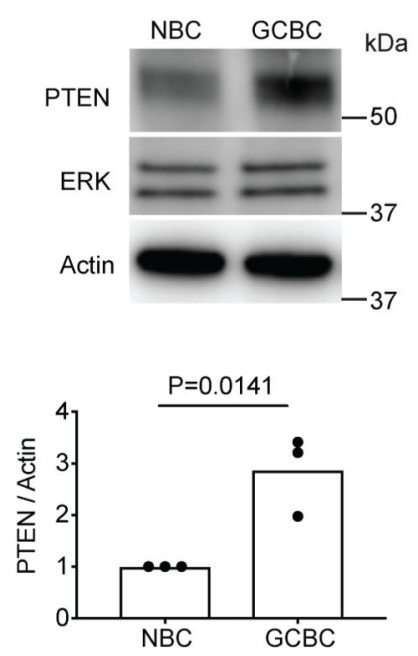

d
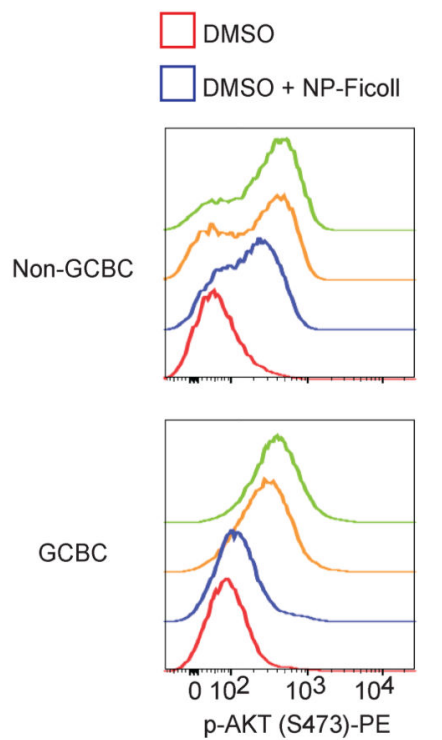

b
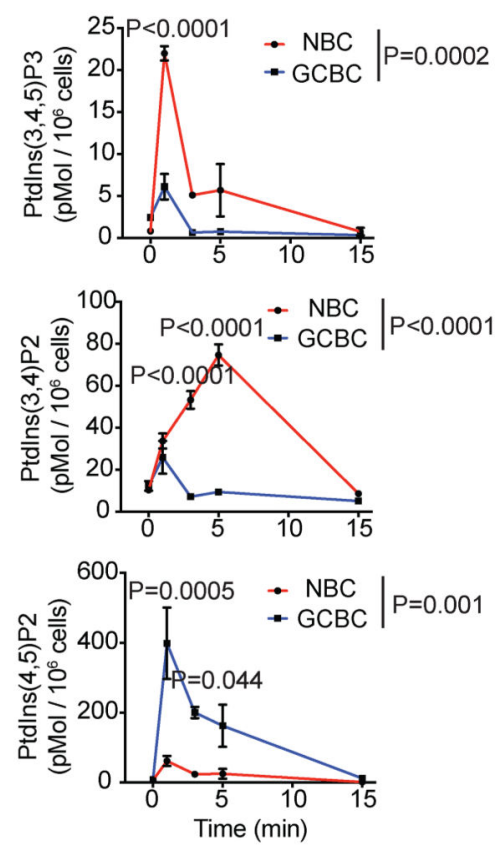
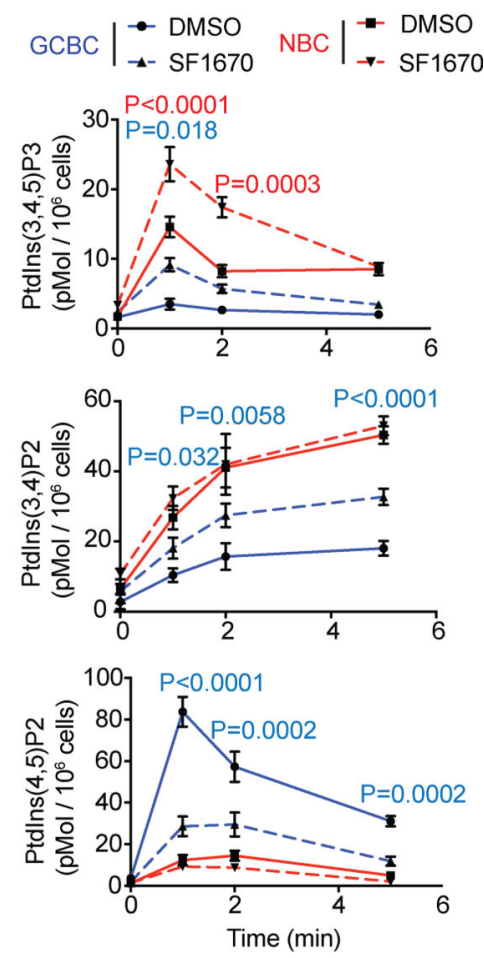

SF1670

SF1670 + NP-Ficoll
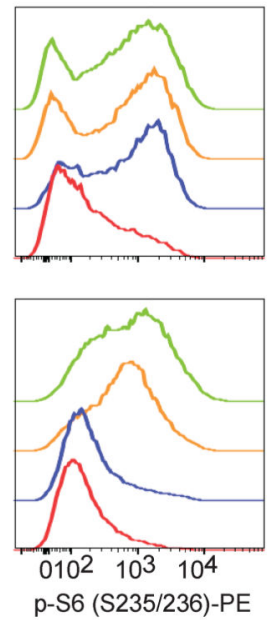

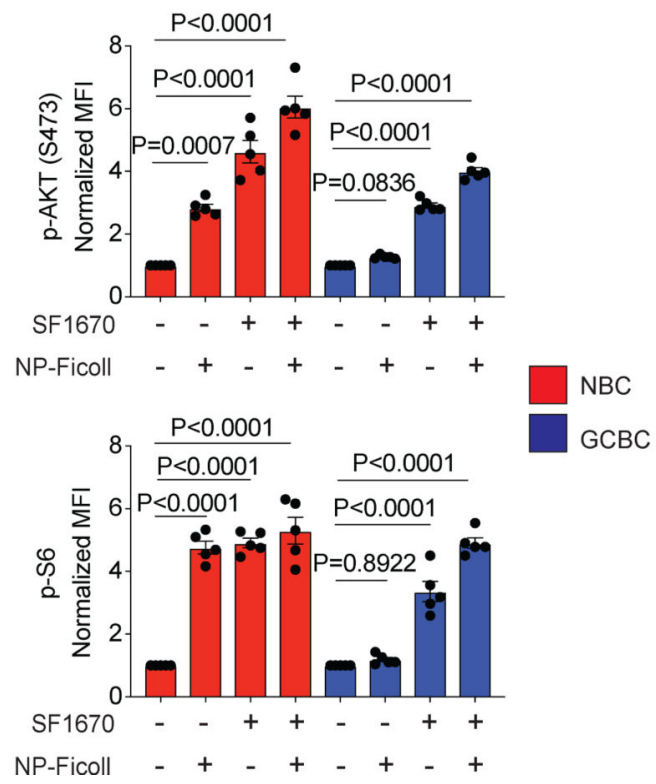

Fig. 2 |. PTEN is highly expressed in GCBCs, controlling phosphatidylinositol phosphate generation and restraining AKT S473 phosphorylation.

a, Protein expression was analyzed by immunoblot with freshly purified B1-8i NBCs and GCBCs; lower graph shows the quantitation of immunoblot data with the mean value. The ratio of PTEN/Actin was normalized to NBCs as 1 . ( $n=3$ from three independent experiments, each sample contain cells pooled from two mice). $P$ values, two-tailed unpaired Student's $t$-test ( $\mathrm{t}=4.161$, d.f.=4). b, Purified MEG NBCs and GCBCs were stimulated with anti-IgM. Inositol lipids from cell lysates were measured by ELISA. Symbols are means and 
error bars are SEM from two independent experiments with cells pooled from five mice per group in each experiment; $P$ values: two-way ANOVA with Sidak's multiple comparisons test $(\operatorname{PtdIns}(3,4,5) \mathrm{P} 3: \mathrm{F}=44.76$, d.f.=4; $\operatorname{PtdIns(3,4)P2:~} \mathrm{F}=33.68$, d.f.=4; $\operatorname{PtdIns(4,5)P2:~}$ $\mathrm{F}=11.31$, d.f.=4). $\mathbf{c}, \mathrm{NBCs}$ and GCBCs from MEG mice ( $\mathrm{n}=2$ except "no inhibitor" condition at 0,1 , and 5 minutes, where $n=3$ )) were treated with the PTEN inhibitor (SF1670) for 10 minutes and then stimulated with anti-IgM. Inositol lipid species were analyzed by ELISA. Cells were pooled from five mice in each experiment. Symbols are means and error bars are SEM. $P$ values compare DMSO and SF1670 treated samples for NBCs (red) and GCBCs (blue) by two-way ANOVA with Sidak's multiple comparisons test (PtdIns(3,4,5)P3: F=76.71, d.f.=3; PtdIns(3,4)P2: F=214.4, d.f.=3; PtdIns(4,5)P2: F=88.16, d.f.=3). d, B1-8i splenocytes were treated with DMSO or SF1670 for 30 minutes before BCR stimulation with NP-Ficoll. Cells were fixed and analyzed by flow cytometry $5 \mathrm{~min}$ (pAKT) or 20 min (p-S6) post stimulation. Right: fold change of MFI, normalized to DMSOalone treated samples. Data are mean \pm SEM from five mice from two independent experiments; $P$ values, one-way ANOVA followed by Turkey's multiple comparisons test (pAKT NBCs: $F=71.93$, d.f.=16; p-AKT GCBCs: $F=262.2$, d.f.=16; p-S6 NBCs: $F=64.86$, d.f.=16; p-S6 GCBCs: F=98.72, d.f.=16). 
a

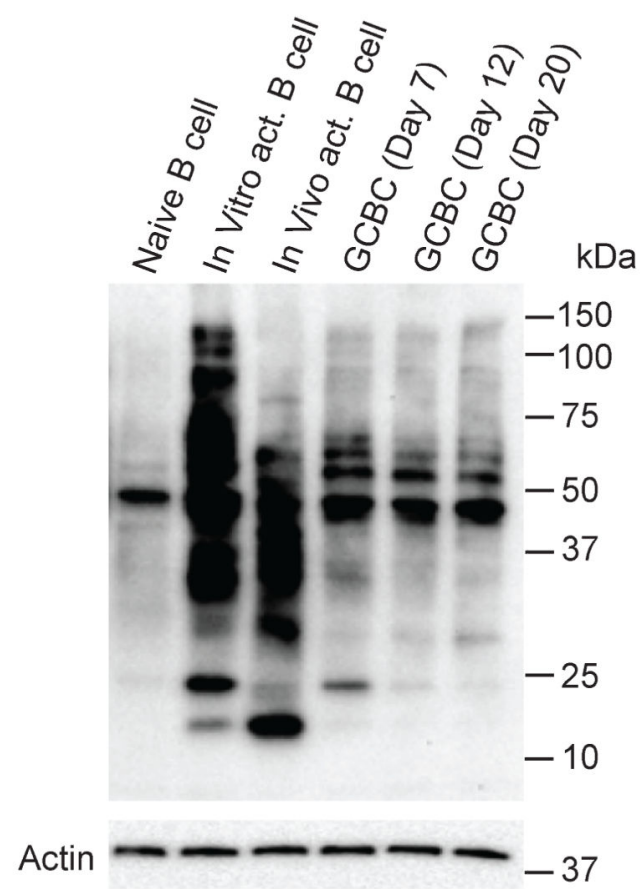

b

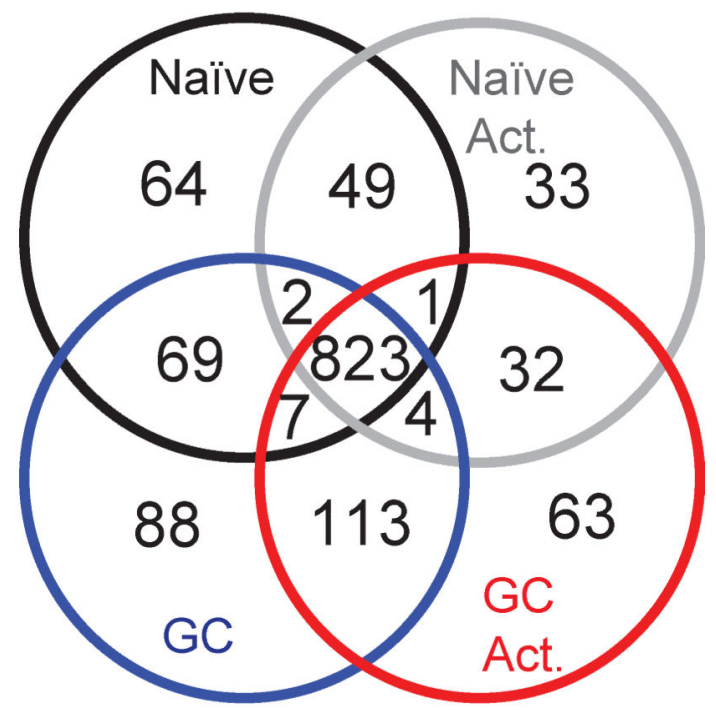

C

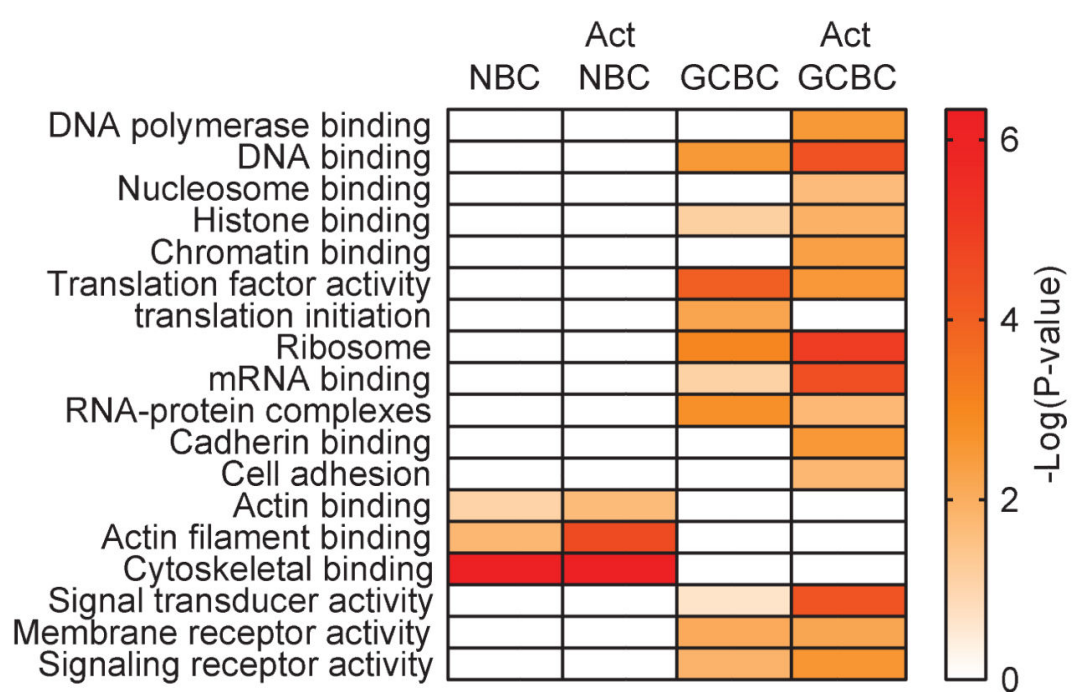

Fig. 3 |. AKT targets different pathways in GCBCs compared to NBCs.

a, B1-8i NBCs, GCBCs from different time points (d7, d12 and d20), in vitro $\mathrm{CpG}$ stimulated B cells, and in vivo NP-Ficoll activated B cells were sorted by FACS. Immunoblot was performed on cell lysates using the AKT phospho-substrate antibody. One representative from two independent experiments is shown; cells were pooled from 4 to 9 mice in each experiment. $\mathbf{b}$, A Venn diagram was constructed to depict the number of AKT phospho-substrates enriched in NBCs, activated NBCs, GCBCs and activated GCBCs using the criteria described in Methods from two independent experiments with cells pooled from three to five mice in each experiment. c, AKT substrates that were specific to NBCs, 
activated NBCs, GCBCs or activated GCBCs were compared to the whole mouse proteome using a binomial test for each gene ontology and pathway term in the PANTHER software package to calculate the probability ( $P$-value) that the number of genes observed in each category occurred by chance. 
a

b

IP: AKT phospho-substrate antibody

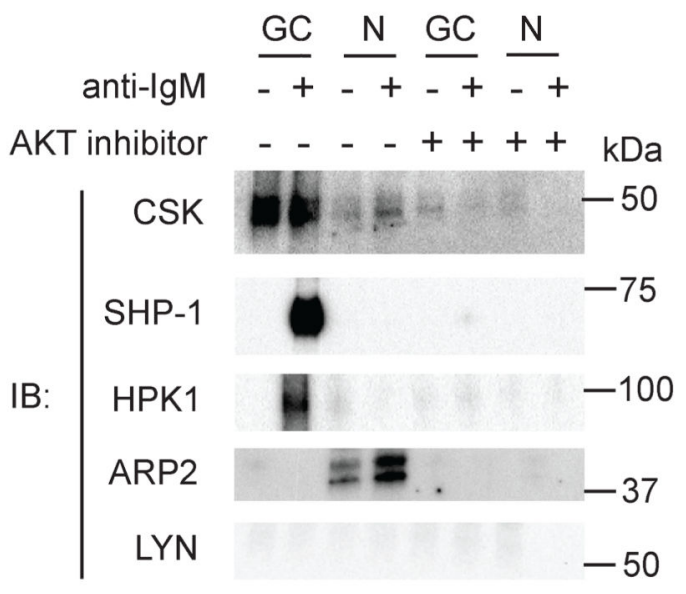

b
- GCBC Unactivated

$\checkmark$ GCBC Activated

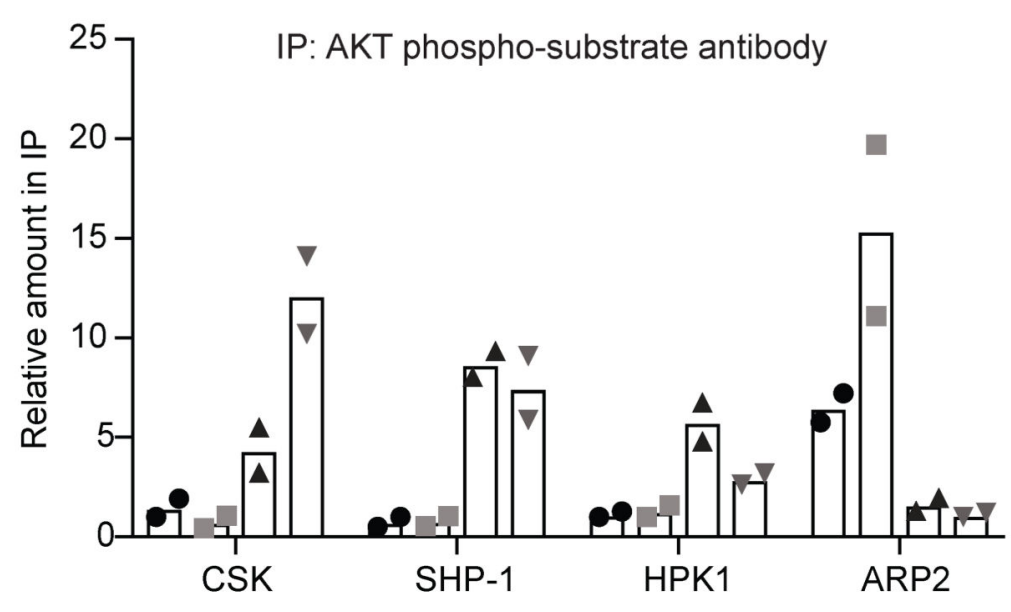

C

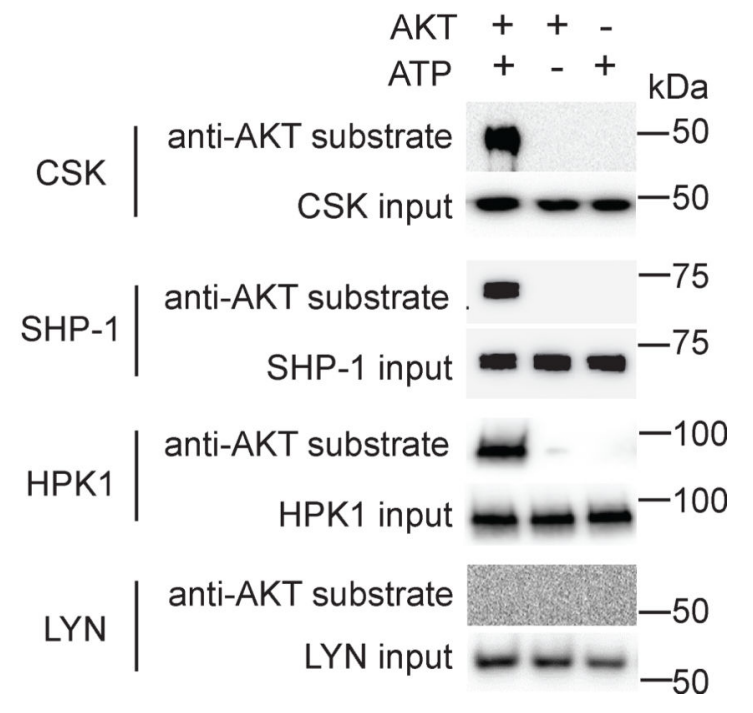

Fig. 4 |. AKT targets proximal BCR signaling regulators in GCBCs.

a, The relative quantitation of proteins of interest immunoprecipitated by bead-conjugated AKT phospho-substrate antibody in Fig. 3 ( $n=2$ from two independent experiments). b, MEG NBCs and GCBCs were pretreated with AKT inhibitor for $10 \mathrm{~min}$ and then stimulated with anti-IgM for 5 min or left unstimulated. Cell lysates were immunoprecipitated by AKT phospho-substrate antibody, and then probed by immunoblot for each of the indicated proteins. Data represent one of two independent experiments. c, In vitro kinase reactions were performed for $30 \mathrm{~min}$ by incubating recombinant pre-activated AKT with CSK, SHP-1, HPK1 or LYN as substrates. Immunoblot using the AKT phospho-substrate antibody was utilized to monitor phosphorylation of the target proteins. A representative of two independent experiments is shown. 
a

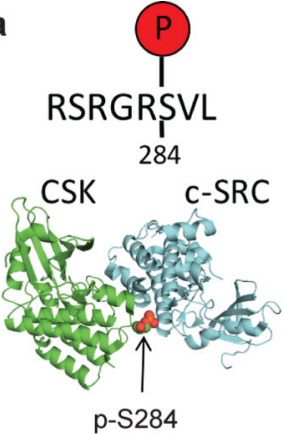

C

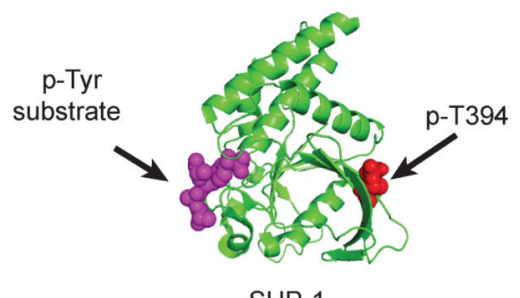

SHP-1

b

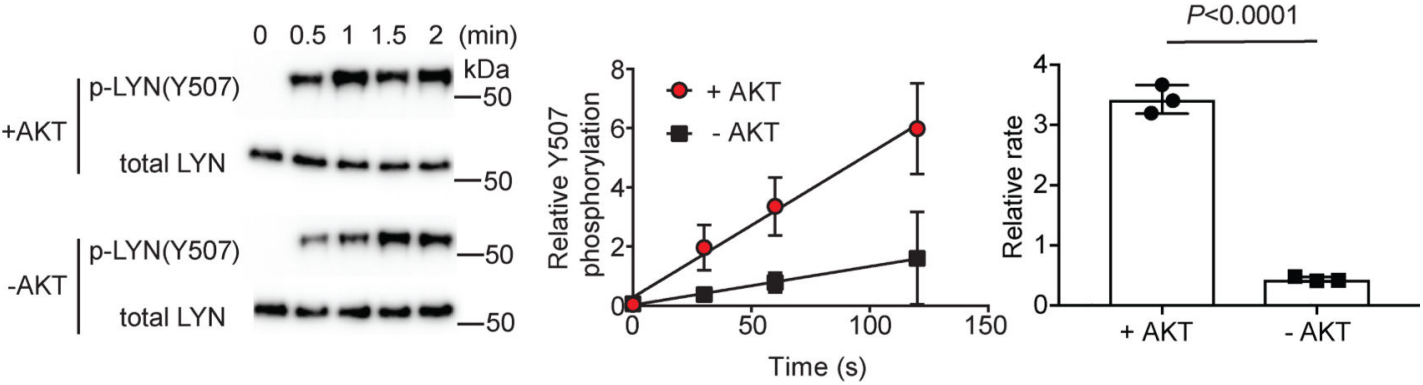

d
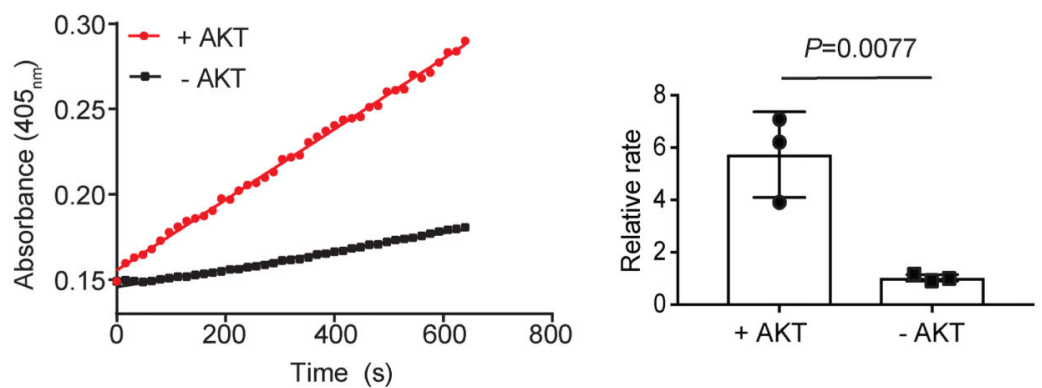

e
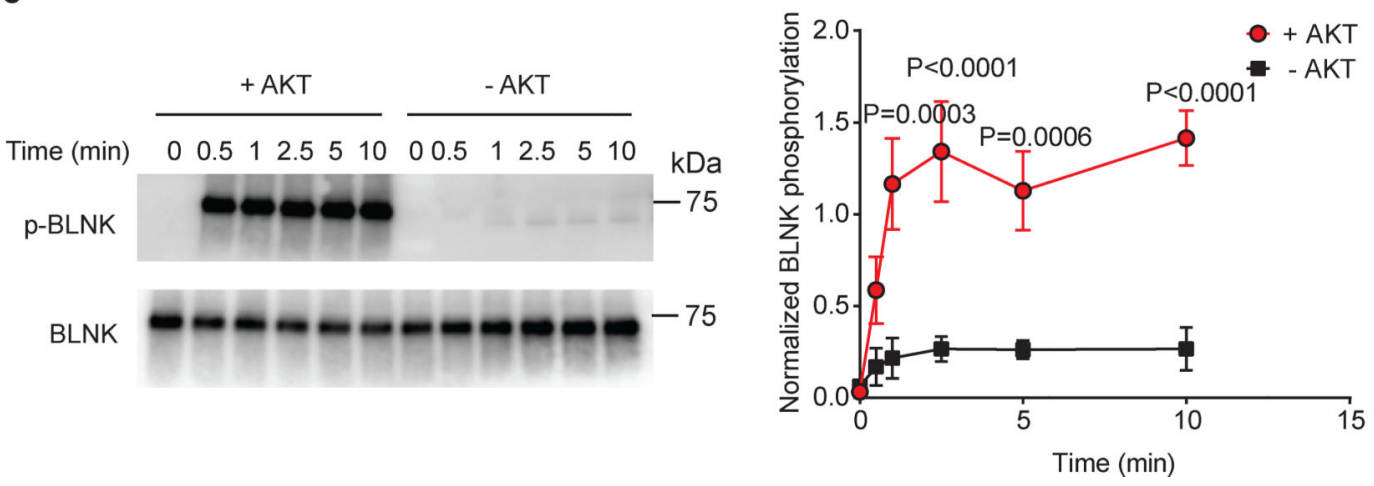

Fig. 5 |. AKT-mediated phosphorylation of CSK, SHP-1 and HPK1 enhances their activity. a, Mass spectrometry was performed on the in vitro kinase reaction of AKT and CSK to identify the AKT phosphorylation site on CSK (S284). S284 phosphorylation was modeled onto the crystal structure of CSK bound to C-SRC (PDB ID 3D7T). b, unphosphorylated CSK or AKT-phosphorylated CSK was reacted with LYN and immunoblot was utilized to monitor LYN Y507 phosphorylation. Densitometry was utilized to quantitate LYN Y507 phosphorylation ( $n=3$ from three independent experiments). The resulting kinetic traces, shown are means \pm SD, were fit with a linear model to determine the relative reaction rate for LYN phosphorylation. $P$ values were derived with a two-tailed Student's $t$-test $(\mathrm{t}=21.55$, d.f.=4). c, Mass spectrometry was utilized to map the AKT phosphorylation site on SHP-1 to 
T394, which is in the catalytic domain. d, A phosphatase assay was performed to measure the activity of unphosphorylated SHP-1 and AKT-phosphorylated SHP-1. The resulting kinetic traces were fit with a linear model to determine the relative reaction rates $(\mathrm{n}=3$ from three independent experiments). Shown are means $\pm \mathrm{SD}, P$ values were calculated with a two-tailed Student's $t$-test (t=4.963, d.f.=4). e, Unphosphorylated HPK1 and AKTphosphorylated HPK1 were incubated with recombinant BLNK and immunoblot was performed with an antibody against phospho-threonine residue. Densitometry was utilized to quantitate BLNK phosphorylation ( $\mathrm{n}=2$ from two independent experiments). Shown are means \pm SD. $P$ values, two-way ANOVA with Sidak's multiple comparisons test $(\mathrm{F}=15.95$, d.f.=5). 
a

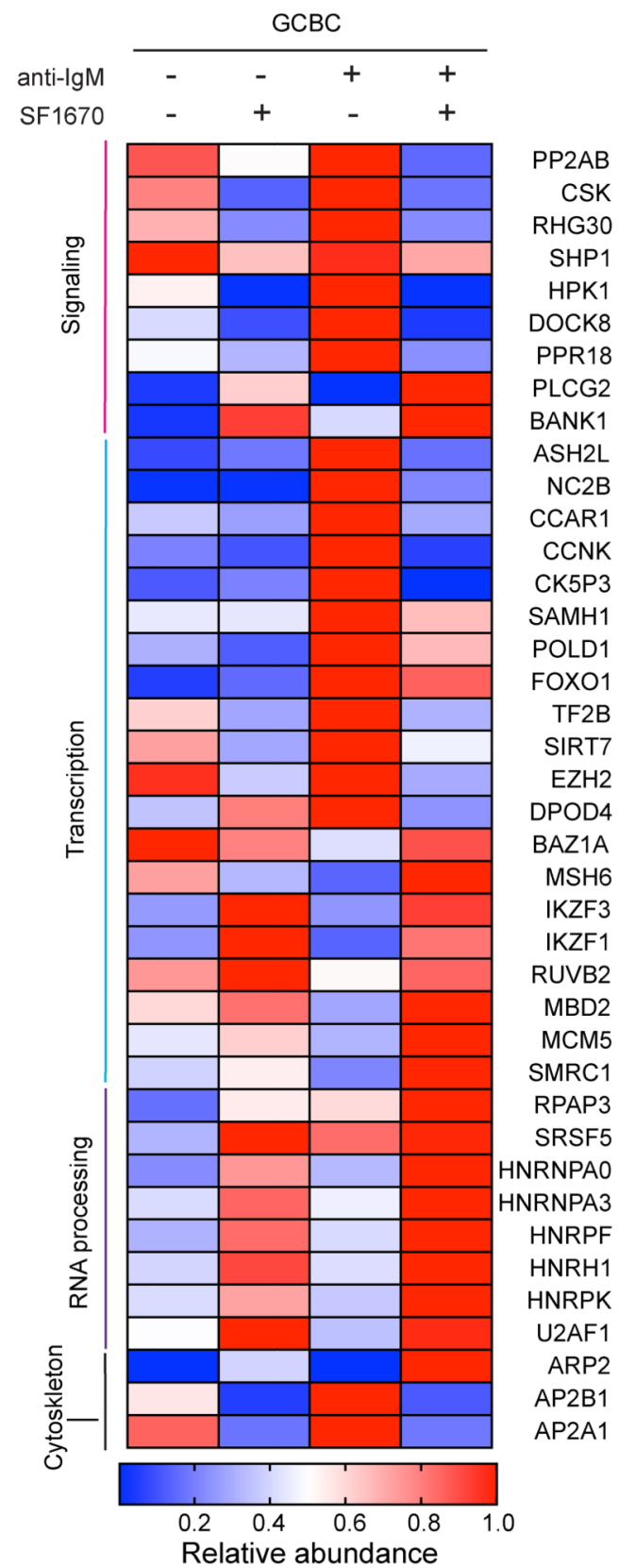

b
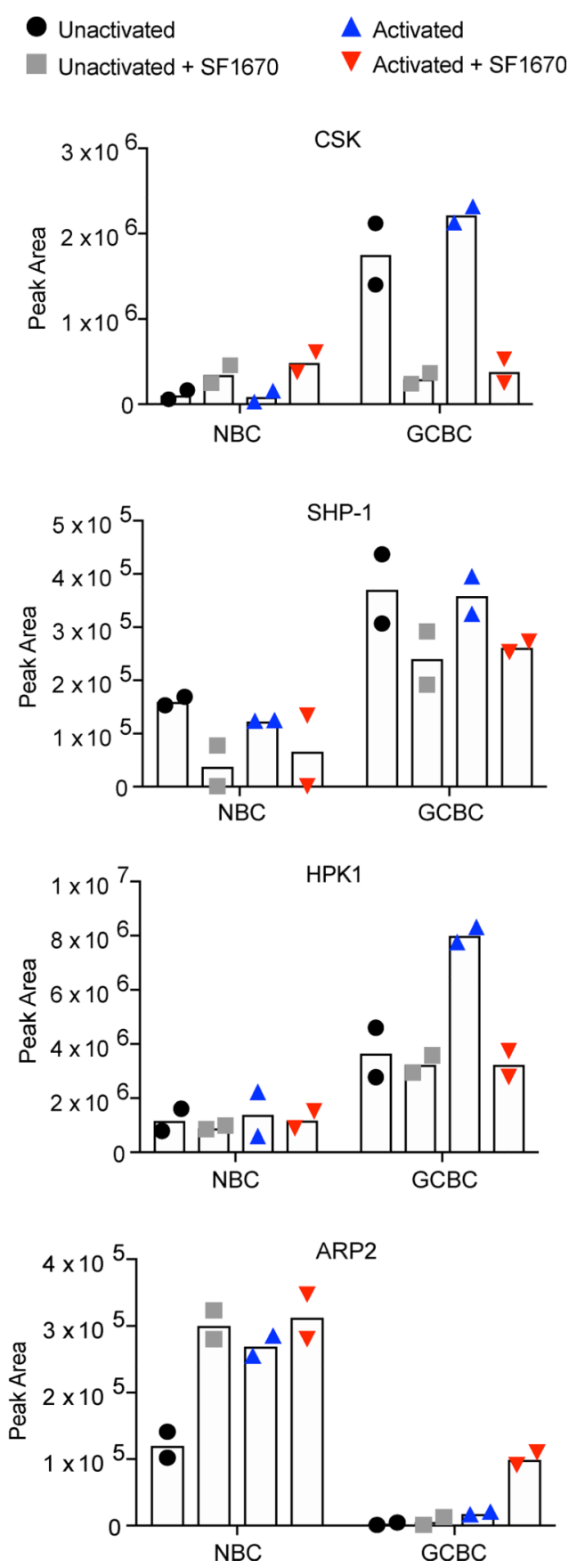

Fig. 6 |. PTEN inhibition alters AKT signaling networks downstream of BCR signaling in GCBCs.

Purified MEG NBCs and GCBCs were incubated with PTEN inhibitor (SF1670) or DMSO for $30 \mathrm{~min}$ and then stimulated with anti-IgM for $5 \mathrm{~min}$. The proteomic study workflow described in Supplementary Fig. 3 was utilized to identify AKT substrates $(n=2$ from two independent experiments). a, The PANTHER software package was utilized to identify pathways targeted by AKT in GCBCs. Heat map shows the relative protein abundance by normalizing the peak area for each experimental group to the maximum peak area observed for each protein. $\mathbf{b}$, The peak area from the mass spectrometric assay was calculated for 
CSK, SHP1, HPK1 and ARP2. Bar graphs show the mean of the results with dots showing results of each independent experiment. 

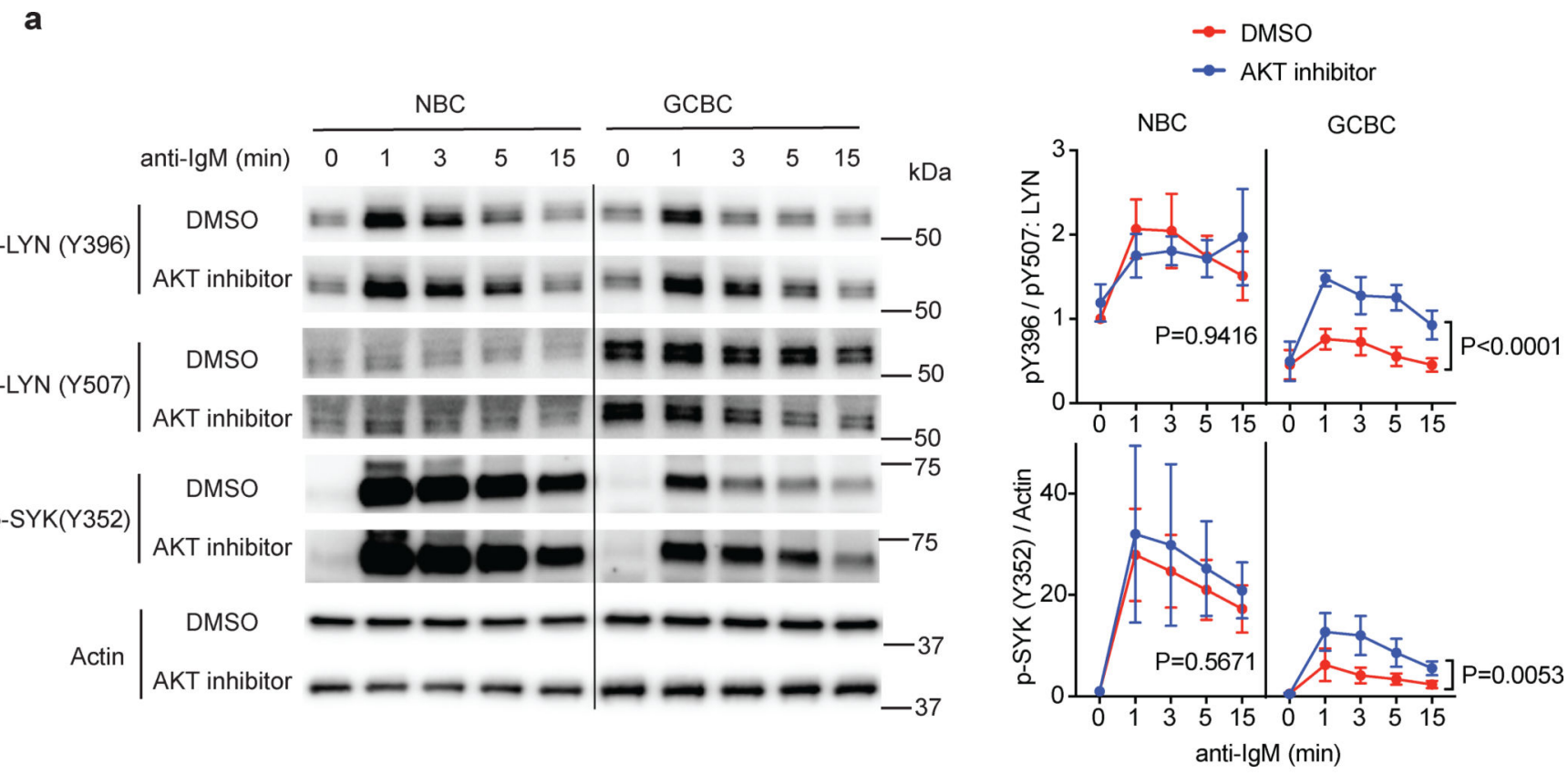

b
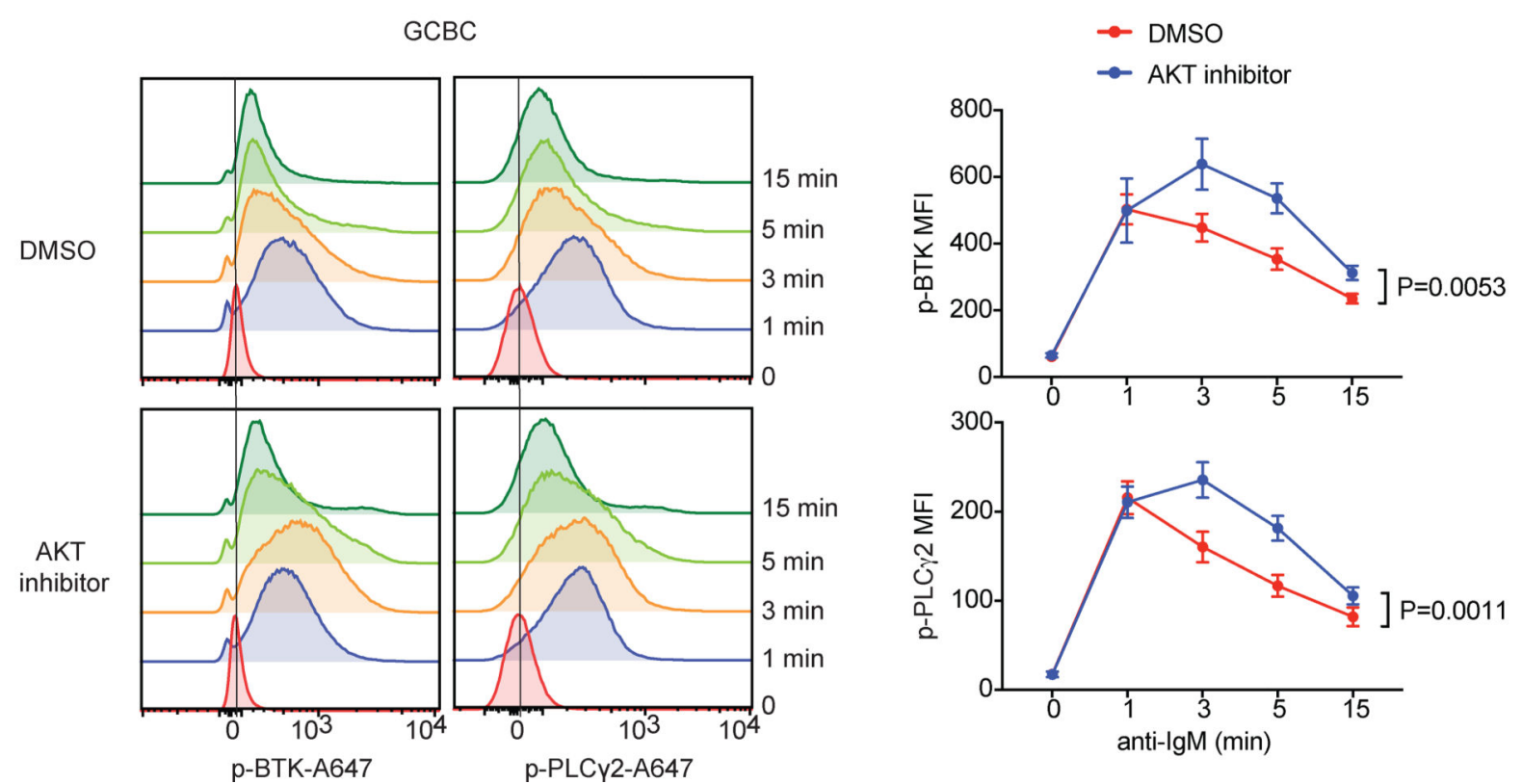

Fig. 7 |. AKT inhibition enhances proximal BCR signaling in GCBCs.

a, Purified NBCs and GCBCs from MEG mice were treated with DMSO or AKT inhibitor for $40 \mathrm{~min}$ before anti-IgM stimulation for the times indicated. Cell lysates were analyzed by immunoblot. Left panel, representative immunoblot of three independent experiments for the p-Tyr species indicated, with cells pooled from 3 to 5 mice in each experiment; right panel, quantitation of immunoblots from all three experiments, the ratios of pY396/pY507 of LYN and p-SYK/Actin were normalized to DMSO treated NBCs (time 0 ) as 1 . Data are mean \pm SEM; $P$ values are comparing treatments (DMSO vs AKT inhibitor) by two-way ANOVA 
(two factors: treatment and time). (pY396/pY507 NBCs: F=0.005502, d.f.=20; pY396/ pY507 GCBCs $F=24.32$, d.f.=20; p-SYK/Actin NBCs: $F=0.3387$, d.f.=20; p-SYK/Actin GCBCs: $F=9.807$, d.f.=20) b, Splenocytes from immunized MEG mice were treated with DMSO or AKT inhibitor for 40 min followed by anti-IgM stimulation for indicated time points. Cells were then analyzed by flow cytometry $(n=4$ from two independent experiments). Representative histogram (left panel) and statistical analysis for MFI of all samples (right panel) are shown for GCBCs. Data are mean \pm SEM; $P$ values are comparing treatments (DMSO vs AKT inhibitor) by two-way ANOVA (two factors: treatment and time). (p-BTK: $F=9.034$, d.f.=30; p-PLC $\gamma 2$ : $F=13.15$, d.f.=30). 